\title{
Testing normality of data on a multivariate grid
}

Article

Accepted Version

Creative Commons: Attribution-Noncommercial-No Derivative Works 4.0

Horváth, L., Kokoszka, P. and Wang, S. (2020) Testing normality of data on a multivariate grid. Journal of Multivariate Analysis, 179. 104640. ISSN 0047-259X doi:

https://doi.org/10.1016/j.jmva.2020.104640 Available at https://centaur.reading.ac.uk/90726/

It is advisable to refer to the publisher's version if you intend to cite from the work. See Guidance on citing.

To link to this article DOI: http://dx.doi.org/10.1016/j.jmva.2020.104640

Publisher: Elsevier

All outputs in CentAUR are protected by Intellectual Property Rights law, including copyright law. Copyright and IPR is retained by the creators or other copyright holders. Terms and conditions for use of this material are defined in the End User Agreement.

\section{www.reading.ac.uk/centaur}

\section{CentAUR}

Central Archive at the University of Reading

Reading's research outputs online 


\title{
Testing Normality of Data on a Multivariate Grid
}

\author{
Lajos Horváth \\ Department of Mathematics \\ University of Utah \\ Salt Lake City \\ UT 84112-0090 \\ Piotr Kokoszka* \\ Department of Statistics \\ Colorado State University \\ Fort Collins \\ CO 80523-1877 \\ Shixuan Wang \\ Department of Economics \\ University of Reading \\ Reading \\ RG6 6AA, United Kingdom
}

${ }^{*}$ Corresponding author. E-mail address: Piotr.Kokoszka@colostate.edu. Declarations of interest: none. 


\begin{abstract}
We propose a significance test to determine if data on a regular $d$-dimensional grid can be assumed to be a realization of Gaussian process. By accounting for the spatial dependence of the observations, we derive statistics analogous to sample skewness and kurtosis. We show that the sum of squares of these two statistics converges to a chi-square distribution with two degrees of freedom. This leads to a readily applicable test. We examine two variants of the test, which are specified by two ways the spatial dependence is estimated. We provide a careful theoretical analysis, which justifies the validity of the test for a broad class of stationary random fields. A simulation study compares several implementations. While some implementations perform slightly better than others, all of them exhibit very good size control and high power, even in relatively small samples. An application to a comprehensive data set of sea surface temperatures further illustrates the usefulness of the test.
\end{abstract}

Keywords: Gaussian process, Lattice data, Significance test, Spatial statistics. 


\section{Introduction}

Nearly all modern spatial statistics applications involve Gaussian processes. While for most large sample results it is not necessary to assume Gaussianity, it is often assumed to improve finite-sample inference and effectively apply Bayesian methods. The same goes for nearly all applications involving conditional and simultaneous autoregressive models in discrete space, see the monographs of Cressie (1993), Stein (1999), Schabenberger \& Gotway (2005), Cressie \& Wikle (2011) and Banerjee et al. (2014). A survey of Gaussian modeling in spatial statistics is given by Gelfand \& Schliep (2016), part III of Gelfand et al. (2010) specifically focuses on methods for discrete spatial data which rely on the Gaussian assumption, and then those that do not. Recent research has focused on applying spatial statistics methods based on the assumption of Gaussianity to large data sets and advancing computational approaches, including parallel and distributed computing, see e.g. Nychka et al. (2015), Paciorek et al. (2015), Katzfuss (2017) and Guhaniyogi \& Banerjee (2018). Methodology and theory for spatial Gaussian models continue to be developed, the references are very numerous. We note the recent work of Stroud et al. (2017), which is concerned with missing values, and of Chang et al. (2019) who study signal identification within the model involving a Gaussian field on a grid.

Despite the prevalence of the assumption of Gaussianity, there appears to exist no significance tests that could be used to assess if it is reasonable to assume that a given spatial data set can be treated as a realization of a Gaussian random field. This is a difficult problem because normality tests, and even exploratory tools like QQ-plots or histograms, require a random sample (iid observations) from a distribution whose Gaussianity is to be determined. For a general spatial data set, testing the joint normality of all finite-dimensional distributions is practically impossible. We will show that it is possible for data defined on a grid under the assumption of stationarity. When the original data do not appear stationary, it is a common practice to attempt to transform them to stationarity. For example, one can use the deformation approach pioneered by Sampson \& Guttorp (1992) and subsequently developed by Schmidt \& O'Hogan (2003), Anderes \& Stein (2008) and Fouedjio et al. (2015), among others. A more common approach is to consider regression models, e.g. Chapter 6 of Schabenberger \& Gotway (2005), whose errors are stationary, and are often assumed jointly normal. These procedures should also be validated by suitable normality tests.

We illustrate an application of our methodology by considering a classical data set of wheat yields studied in some detail in Section 4.5 of Cressie (1993), and many earlier papers cited there. The data are shown in Figure 1. It is argued in Cressie (1993) that no transformation of these data is needed to ensure stationarity. The question we want to answer is if these data can be considered to be a realization of a Gaussian process, i.e. if these values can be assumed to be a realization of a random field $X_{i, j}, i, j \in \mathbb{Z}$, whose all finite-dimesional distributions are multivariate normal. This question is difficult to answer 


\section{North}

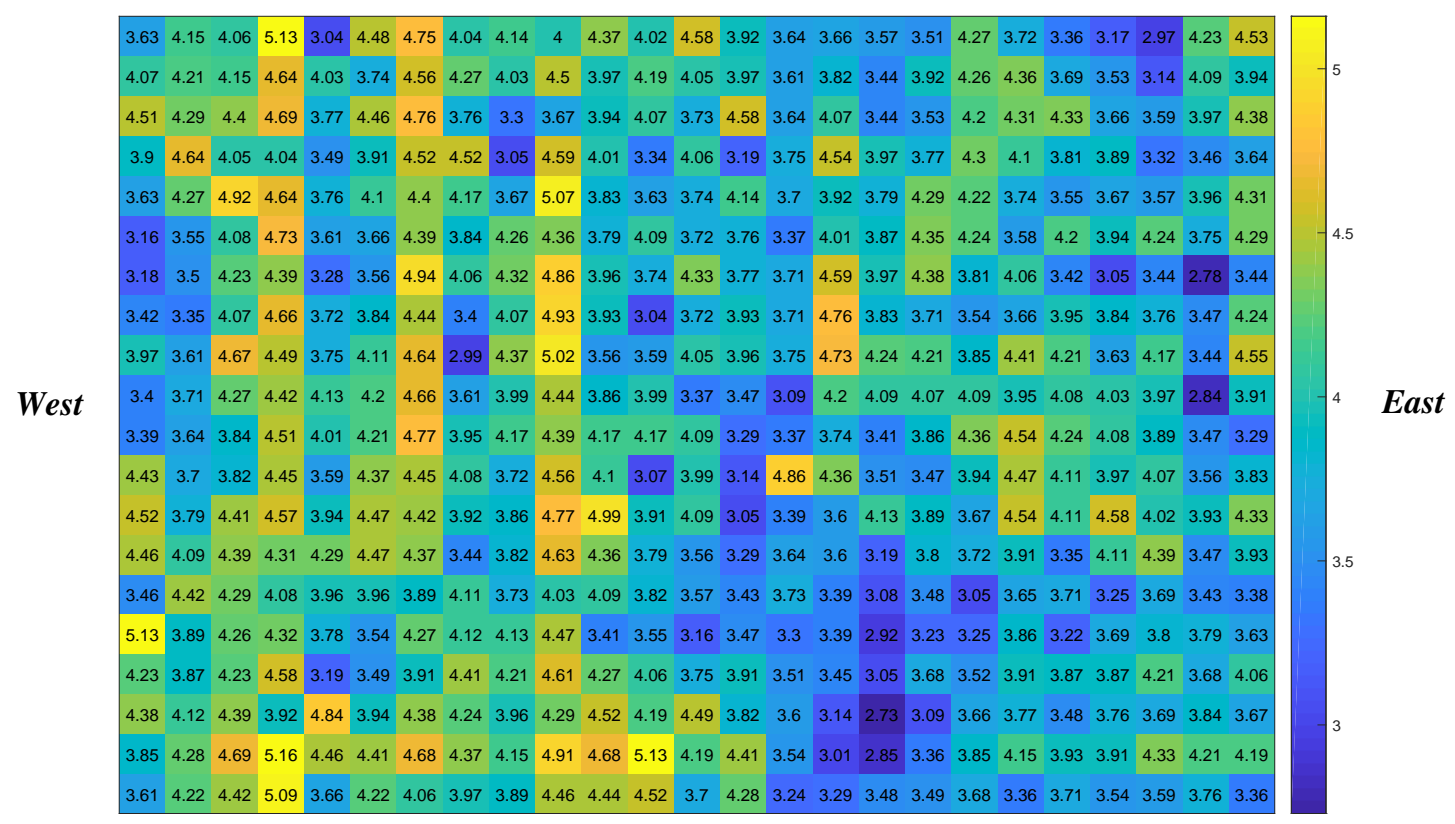

South

Figure 1: Mercer and Hall wheat-yield data

because the pronounced spatial dependence of these data can "force" more large or small values in a finite region than univariate normality might suggest. More fundamentally, since these data are not a random sample, usual exploratory plots or tests cannot be relied on. Our significance test shows that these data can be assumed to be a realization of a Gaussian process. Depending on the implementation, the $\mathrm{P}$-values are between $16 \%$ and $52 \%$, details are shown in Section I of the online supplement. Our simulations shows that most implementations have sufficient power to detect a departure from normality that matters, even for the relatively small sample size $(20 \times 25$ grid $)$ of the data in Figure 1 .

We hope that the test we propose will turn out to be a useful diagnostic tool, which may lend confidence in the application of various methodologies based on the normality assumption, or provide a caution on the validity of conclusions. An appealing feature of our test is that the test statistics can be computed fairly easily using existing $R$ or MATLAB software, and the critical values are those of a chi-square distribution. The test has good empirical size and power, and can be justified asymptotically using recent advances in the asymptotic theory for random fields and new arguments related to the quantification of spatial dependence.

The assumption of normality has underlain much of the development of statistics, well beyond spatial statistics, and many tests have been proposed. Perhaps the best 
known is the Shapiro \& Wilk (1965) test, which has been extended and improved in many directions, Royston (1982, 1983, 1992). Tests based on the empirical distribution function have also been extensively used, Anderson \& Darling (1954), Stephens (1974), Scholz \& Stephens (1997). Great many other approaches have been proposed, Mardia (1970, 1974), D’Agostino et al. (1990), Henze \& Zirkler (1990), Doornik \& Hansen (2008), among many others. However, perhaps the most commonly used test is the Jarque and Bera $(1980,1987)$ test. It checks if the first four moments of a distribution agree with the those of a normal distribution. This is a general direction we take. Our test will not detect very subtle departures from normality, which manifest themselves in discrepancy in moments beyond the first four, but it will detect most commonly encountered deviations from normality.

The paper is organized as follows. In Section 2 we develop the test. Its finite sample performance is evaluated in Section 3 by means of a simulation study and an application to a climate data set. There are many possible implementations of our general paradigm, which must be evaluated and compared. The proofs of the mathematical results of Section 2, needed to derive and justify the test, are presented in Section II of an online supplement, which also contains additional details of the test procedure and additional tables, which support our conclusions and recommendations.

\section{Testing procedure and its large sample justification}

We derive and formulate the testing procedure in Section 2.1, where we also specify the most important assumptions for its validity. A fundamental ingredient of our approach is the quantification and estimation of spatial dependence, this is treated in Section 2.2. Asymptotic theory underlying both Sections 2.1 and 2.2 is developed in Section 2.3.

\subsection{Assumptions and test derivation}

Let $\mathbb{Z}^{d}$ denote the set of $d$-dimensional vectors with integer coordinates. We assume that the observations $X_{i}$ follow the model

$$
X_{i}=\mu+e_{i}, \quad i \in \mathbb{Z}^{d}
$$

where $\left\{e_{i}\right\}$ is a strictly stationary, zero mean spatial process. The mean $\mu$ is unknown.

We want to test

$H_{0}$ : the $X_{i}$ are jointly normal,

against the alternative that $H_{0}$ does not hold. The test is based on observations $X_{i}$, $i \in \Gamma_{n} \subset \mathbb{Z}^{d}$. The domain $\Gamma_{n}$ is indexed by positive integers $n$, which are not sample sizes, but sample indexes in increasing domain asymptotics. The sample size is denoted by $n_{\Gamma}$, the cardinality of the set $\Gamma_{n}, n_{\Gamma}=\left|\Gamma_{n}\right|$. If $d=2$, and $\Gamma_{n}=\Gamma_{N, M}:=$ 
$\{(i, j), 1 \leq i \leq N, 1 \leq j \leq M\}$, then $n_{\Gamma}=N M$. Let $\partial \Gamma_{n}$ denote the boundary of $\Gamma_{n}$ and $\left|\partial \Gamma_{n}\right|$ its cardinality. We assume that, as $n \rightarrow \infty$,

$$
\frac{\left|\partial \Gamma_{n}\right|}{n_{\Gamma}} \rightarrow 0
$$

Condition (2.1) states that asymptotically there should be many more points in the interior of the domain than at its boundary. If $d=2$, and $\Gamma_{n}=\Gamma_{N, M}$, defined above, then (2.1) holds if and only of $\min (N, M) \rightarrow \infty$.

We assume that under the null hypothesis $\left\{e_{i}\right\}$ is a Gaussian spatial linear process, i.e. it satisfies the following assumption.

Assumption 2.1 The $e_{i}$ are spatial moving averages,

$$
e_{i}=\sum_{s \in \mathbb{Z}^{d}} a_{s} \varepsilon_{i-s}, \quad i \in \mathbb{Z}^{d}
$$

with independent, standard normal innovations $\varepsilon_{i}$, and the coefficients $a_{s}$ satisfying

$$
\sum_{s \in \mathbb{Z}^{d}}\left|a_{s}\right|<\infty
$$

Assumption 2.1 implies that the field $\left\{X_{i}\right\}$ is strictly stationary and Gaussian, with spatial dependence quantified by conditions (2.2) and (2.3). Linearity in (2.2) is needed to ensure normality of the observations. The summability condition in (2.3) cannot be relaxed because the required CLT would not hold with standard rate, see Lahiri \& Robinson (2016). Under Assumption 2.1, the random variables

$$
z_{i}=\frac{X_{i}-\mu}{\sigma}, \text { with } \sigma^{2}=\sum_{s \in \mathbb{Z}^{d}} a_{s}^{2},
$$

are standard normal (but, in general, not independent). The $z_{i}$ must be approximated by random variables that can be computed from the sample. For this purpose, define

$$
S_{n}^{2}=\frac{1}{n_{\Gamma}} \sum_{i \in \Gamma_{n}}\left(X_{i}-\bar{X}_{n}\right)^{2}, \quad \bar{X}_{n}=\frac{1}{n_{\Gamma}} \sum_{i \in \Gamma_{n}} X_{i} .
$$

Our tests statistics are based on the standardized observations

$$
x_{i}=x_{i, n}=\frac{X_{i}-\bar{X}_{n}}{S_{n}}, \quad i \in \Gamma_{n},
$$

which are sample counterparts of the standard normal $z_{i}$ defined above. Using the $x_{i}$, we define the sample skewness and kurtosis by

$$
\mathcal{S}_{n}=\frac{1}{n_{\Gamma}^{1 / 2}} \sum_{i \in \Gamma_{n}} x_{i}^{3} \quad \text { and } \quad \mathcal{K}_{n}=\frac{1}{n_{\Gamma}^{1 / 2}} \sum_{i \in \Gamma_{n}}\left(x_{i}^{4}-3\right) .
$$


As we will see in Section 2.3, the asymptotic variances of $\mathcal{S}_{n}$ and $\mathcal{K}_{n}$ are, respectively,

$$
\phi_{\mathcal{S}}^{2}=\sum_{i \in \mathbb{Z}^{d}} E\left[\left(z_{0}^{3}-3 z_{0}\right)\left(z_{i}^{3}-3 z_{i}\right)\right]
$$

and

$$
\phi_{\mathcal{K}}^{2}=\sum_{i \in \mathbb{Z}^{d}} E\left[\left(z_{0}^{4}-6 z_{0}^{2}+3\right)\left(z_{i}^{4}-6 z_{i}^{2}+3\right)\right] .
$$

In particular,

$$
\phi_{\mathcal{K}}^{2} \neq \sum_{i \in \mathbb{Z}^{d}} E\left[\left(z_{0}^{4}-3\right)\left(z_{i}^{4}-3\right)\right] .
$$

This motivates the introduction of modified sample skewness and kurtosis defined by

$$
\mathcal{S}_{n}^{\star}=\frac{1}{n_{\Gamma}^{1 / 2}} \sum_{i \in \Gamma_{n}}\left(x_{i}^{3}-3 x_{i}\right) \quad \text { and } \quad \mathcal{K}_{n}^{\star}=\frac{1}{n_{\Gamma}^{1 / 2}} \sum_{i \in \Gamma_{n}}\left(x_{i}^{4}-6 x_{i}^{2}+3\right) .
$$

Observe that $\mathcal{S}_{n}^{\star}=\mathcal{S}_{n}$ because $\sum_{i \in \Gamma_{n}} x_{i}=0$. The statistics $\mathcal{S}_{n}^{\star}$ and $\mathcal{K}_{n}^{\star}$ also have asymptotic variances, respectively, $\phi_{\mathcal{S}}^{2}$ and $\phi_{\mathcal{K}}^{2}$, and are better matched to them in finite samples because $\phi_{\mathcal{S}}^{2}$ and $\phi_{\mathcal{K}}^{2}$ are direct counterparts of spatial long-run variances of the sequences $\left\{x_{i}^{3}-3 x_{i}\right\}$ and $\left\{x_{i}^{4}-6 x_{i}^{2}+3\right\}$.

Denoting by $\hat{\phi}_{\mathcal{S}}$ and $\hat{\phi}_{\mathcal{K}}$ consistent estimators of $\phi_{\mathcal{S}}$ and $\phi_{\mathcal{K}}$, the test statistic is defined as

$$
J_{n}^{\star}=\frac{\mathcal{S}_{n}^{\star 2}}{\hat{\phi}_{\mathcal{S}}^{2}}+\frac{\mathcal{K}_{n}^{\star 2}}{\hat{\phi}_{\mathcal{K}}^{2}} .
$$

It is the sum of squares of normalized skewness and kurtosis. As will be stated in Section $2.3, J_{n}^{\star}$ is asymptotically chi-square with two degrees of freedom. The test thus is:

Reject $H_{0}$ at significance level $\alpha$ if $J_{n}^{\star}>\chi_{2}^{2}(1-\alpha)$, where $\chi_{2}^{2}(1-\alpha)$ is the $(1-\alpha)$ th quantile of the chi-square distribution with two degrees of freedom.

Suitable estimators $\hat{\phi}_{\mathcal{S}}^{2}$ and $\hat{\phi}_{\mathcal{K}}^{2}$ are derived in Section 2.2, see formulas (2.12) and (2.13).

The key to understanding the need for the modified kurtosis is the fact that

$$
\phi_{\mathcal{K}}^{2} \neq \sum_{i \in \mathbb{Z}^{d}} E\left[\left(z_{0}^{4}-3\right)\left(z_{i}^{4}-3\right)\right] .
$$

Formula (2.8) must be used instead. Next notice that $\phi_{\mathcal{K}}^{2}$ given by (2.8) is the long-run variance of the unobservable field $\left\{z_{i}^{4}-6 z_{i}^{2}+3\right\}$. We replace the $z_{i}$ by the observable $x_{i}$, which approximate them with an asymptotically negligible effect. In particular, $\operatorname{Var}\left[\mathcal{K}_{n}^{\star}\right]=$ $\phi_{\mathcal{K}}^{2}$, so $\mathcal{K}_{n}^{\star 2}$ divided by an estimator of the variance of $\mathcal{K}_{n}^{\star}$ is a Wald statistic, which is asymptotically $\chi_{1}^{2}$. (The population kurtosis is zero under the null hypothesis.) The same argument applies the skewness. We show that these two components are asymptotically independent, so their sum is asymptotically $\chi_{2}^{2}$. 


\subsection{Estimation of the spatial long run variances}

It is useful to consider a more general setting. Suppose $\left\{y_{i}, i \in \mathbb{Z}^{d}\right\}$ is a zero mean strictly stationary scalar random field such that $E y_{0}^{2}<\infty$, whose covariances are $\gamma(j)=$ $E\left[y_{0} y_{j}\right], j \in \mathbb{Z}^{d}$. The objective is to estimate the long-run, or asymptotic, variance defined by

$$
\sigma^{2}=\sum_{j \in \mathbb{Z}^{d}} \gamma(j)=\sum_{j \in \mathbb{Z}^{d}} E\left[y_{0} y_{j}\right]
$$

We assume throughout that

$$
\sum_{j \in \mathbb{Z}^{d}}|\gamma(j)|<\infty
$$

so that $\sigma^{2}$ can be defined. We observe $y_{j} \in \Gamma_{n}$, which is a rectangle whose all dimensions are increasing, as specified in the following assumption.

Assumption 2.2 The spatial domain $\Gamma_{n}$ is given by

$$
\Gamma_{n}=\left\{1,2, \ldots, n_{1}\right\} \times\left\{1,2, \ldots, n_{2}\right\} \times \ldots \times\left\{1,2, \ldots, n_{d}\right]
$$

and $n^{\star}:=\min _{1 \leq i \leq d} n_{i} \rightarrow \infty$.

The sample covariances are defined by

$$
\hat{\gamma}(j)=\left|\Gamma_{n}(j)\right|^{-1} \sum_{i \in \Gamma_{n}(j)} y_{i} y_{i+j}, \quad \text { where } \Gamma_{n}(j)=\left\{i \in \Gamma_{n}: i+j \in \Gamma_{n}\right\} .
$$

To provide explicit formulas, in the following we use the notation $j=\left(j_{1}, \ldots, j_{d}\right)$. In this setting, $\sigma^{2}$ is estimated by the kernel estimator

$$
\hat{\sigma}_{n}^{2}=\sum_{\ell=1}^{d} \sum_{\left|j_{\ell}\right| \leq n_{\ell}}\left\{\prod_{\ell=1}^{d} K\left(\frac{j_{\ell}}{h_{\ell}}\right)\right\} \hat{\gamma}\left(j_{1}, \ldots, j_{d}\right)
$$

where $K$ is a univariate kernel satisfying the following commonly used assumption.

Assumption 2.3 The kernel $K$ is a continuous function on the interval $[-1,1]$ satisfying $K(0)=1$. The bandwidths $h_{\ell}$ satisfy $h^{\star}:=\max _{1 \leq \ell \leq d} h_{\ell} \rightarrow \infty$, as $n \rightarrow \infty$.

In our context, we use estimator (2.11) computed from $y_{i}=x_{i}^{3}-3 x_{i}$ and $y_{i}=x_{i}^{4}-$ $6 x_{i}+3$. These $y_{i}$ do not form a strictly stationary random field. Due to the random normalization in (2.5), they form a structure which could be called a spatial triangular array. However, the $z_{i}$ defined by (2.4) do form a strictly stationary random field, so it must be shown that replacing the $x_{i}$ by the $z_{i}$ introduces an asymptotically negligible 
effect into the estimation of $\phi_{\mathcal{S}}^{2}$ and $\phi_{\mathcal{K}}^{2}$. This will be established in the proof of Theorem 2.2. We first introduce the required notation. Set

$$
y_{i}^{\mathcal{S}}=x_{i}^{3}-3 x_{i}, \quad y_{i}^{\mathcal{K}}=x_{i}^{4}-6 x_{i}+3
$$

and

$$
\bar{y}_{\mathcal{S}}=\frac{1}{n_{\Gamma}} \sum_{i \in \Gamma_{n}} y_{i}^{\mathcal{S}}, \quad \bar{y}_{\mathcal{K}}=\frac{1}{n_{\Gamma}} \sum_{i \in \Gamma_{n}} y_{i}^{\mathcal{K}} .
$$

Next, we define the sample covariances

$$
\begin{aligned}
& \hat{\gamma}_{\mathcal{S}}(j)=\left|\Gamma_{n}(j)\right|^{-1} \sum_{i \in \Gamma_{n}(j)}\left(y_{i}^{\mathcal{S}}-\bar{y}_{\mathcal{S}}\right)\left(y_{i+j}^{\mathcal{S}}-\bar{y}_{\mathcal{S}}\right) \\
& \hat{\gamma}_{\mathcal{K}}(j)=\left|\Gamma_{n}(j)\right|^{-1} \sum_{i \in \Gamma_{n}(j)}\left(y_{i}^{\mathcal{K}}-\bar{y}_{\mathcal{K}}\right)\left(y_{i+j}^{\mathcal{K}}-\bar{y}_{\mathcal{K}}\right) .
\end{aligned}
$$

Using notation

$$
\sum_{j \in J(h)} w_{h}(j) g(j)=\sum_{\ell=1}^{d} \sum_{\left|j_{\ell}\right| \leq n_{\ell}}\left\{\prod_{\ell=1}^{d} K\left(\frac{j_{\ell}}{h_{\ell}}\right)\right\} g\left(j_{1}, \ldots, j_{d}\right),
$$

which applies to any function $g$ on $\mathbb{Z}^{d}$, we define the kernel estimators

$$
\hat{\phi}_{\mathcal{S}, \text { kern }}^{2}=\sum_{j \in J(h)} w_{h}(j) \hat{\gamma}_{\mathcal{S}}(j), \quad \hat{\phi}_{\mathcal{K}, \text { kern }}^{2}=\sum_{j \in J(h)} w_{h}(j) \hat{\gamma}_{\mathcal{K}}(j) .
$$

The idea behind the kernel estimators is as follows. Focus on $\hat{\phi}_{\mathcal{K} \text {,kern }}^{2}$ and consult formula (2.8). We replace the model autocovariances $E\left[\left(z_{0}^{4}-6 z_{0}^{2}+3\right)\left(z_{j}^{4}-6 z_{j}^{2}+3\right)\right]$ by the sample autocovariances $\hat{\gamma}_{\mathcal{K}}(j)$. The latter are variable if the set $\Gamma_{n}(j)$ is small, i.e. if $j$ is "spatially large". For this reason, we put smaller weights on them. This idea has been commonly used in time series analysis.

Another class of estimators can be derived as follows. Set $\rho_{i}=E\left[z_{0} z_{i}\right]$. Tedious calculations, using the values of the moments of the standard normal distributions, show that

$$
\phi_{\mathcal{S}}^{2}=6 \sum_{i \in \mathbb{Z}^{d}} \rho_{i}^{3} \quad \text { and } \quad \phi_{\mathcal{K}}^{2}=24 \sum_{i \in \mathbb{Z}^{d}} \rho_{i}^{4}
$$

We estimate the $\rho_{i}$ by the sample covariances of the $x_{i}$, i.e. by (recall that $\bar{x}=0$ )

$$
\hat{\gamma}_{x}(j)=\left|\Gamma_{n}(j)\right|^{-1} \sum_{i \in \Gamma_{n}(j)} x_{i} x_{i+j}
$$

and define the power estimators

$$
\hat{\phi}_{\mathcal{S}, \text { pow }}^{2}=6 \sum_{j \in J(h)} w_{h}(j) \hat{\gamma}_{x}^{3}(j), \quad \hat{\phi}_{\mathcal{K}, \text { pow }}^{2}=24 \sum_{j \in J(h)} w_{h}(j) \hat{\gamma}_{x}^{4}(j),
$$


i.e.

$$
\begin{aligned}
& \hat{\phi}_{\mathcal{S}, \text { pow }}^{2}=6 \sum_{\ell=1}^{d} \sum_{\left|j_{\ell}\right| \leq h_{\ell}}\left\{\prod_{\ell=1}^{d} K\left(\frac{j_{\ell}}{n_{\ell}}\right)\right\} \hat{\gamma}_{x}^{3}\left(j_{1}, \ldots, j_{d}\right), \\
& \hat{\phi}_{\mathcal{K}, \text { pow }}^{2}=24 \sum_{\ell=1}^{d} \sum_{\left|j_{\ell}\right| \leq h_{\ell}}\left\{\prod_{\ell=1}^{d} K\left(\frac{j_{\ell}}{n_{\ell}}\right)\right\} \hat{\gamma}_{x}^{4}\left(j_{1}, \ldots, j_{d}\right) .
\end{aligned}
$$

The consistency of the above spatial long-run variance estimators is established in Section 2.3. More explicit formulas for the commonly encountered case of a 2D rectangular domain are given in Section III of the Supplement.

\subsection{Asymptotic theory}

This section contains asymptotic results, which justify the application of the test for a large class of stationary fields. All proofs are given in Section II of the supplement. The first result establishes the asymptotic distribution of the sample skewness $\mathcal{S}_{n}$ and kurtosis $\mathcal{K}_{n}$, and their modified versions $\mathcal{S}_{n}^{\star}$ and $\mathcal{K}_{n}^{\star}$. Very little must be assumed about the shape of the spatial domain $\Gamma_{n}$.

Theorem 2.1 Suppose condition (2.1) and Assumption 2.1 hold. Then the series (2.7) and (2.8) defining, respectively, $\phi_{\mathcal{S}}^{2}$ and $\phi_{\mathcal{K}}^{2}$ are absolutely convergent, and the vectors $\left[\mathcal{S}_{n}, \mathcal{K}_{n}\right]^{\top}$ and $\left[\mathcal{S}_{n}^{\star}, \mathcal{K}_{n}^{\star}\right]^{\top}$ both converge to the bivariate normal distribution with mean zero and covariance matrix

$$
\left[\begin{array}{cc}
\phi_{\mathcal{S}}^{2} & 0 \\
0 & \phi_{\mathcal{K}}^{2}
\end{array}\right]
$$

Based on Theorem 2.1, we consider the test statistics

$$
\widehat{J}_{n}=\frac{\mathcal{S}_{n}^{2}}{\hat{\phi}_{\mathcal{S}}^{2}}+\frac{\mathcal{K}_{n}^{2}}{\hat{\phi}_{\mathcal{K}}^{2}} \quad \text { and } \quad J_{n}^{\star}=\frac{\mathcal{S}_{n}^{\star 2}}{\hat{\phi}_{\mathcal{S}}^{2}}+\frac{\mathcal{K}_{n}^{\star 2}}{\hat{\phi}_{\mathcal{K}}^{2}} .
$$

The following corollary is an immediate consequence of Theorem 2.1.

Corollary 2.1 Suppose condition (2.1) and Assumption 2.1 hold, and

$$
\hat{\phi}_{\mathcal{S}}^{2} \stackrel{P}{\rightarrow} \phi_{\mathcal{S}}^{2} \quad \text { and } \quad \hat{\phi}_{\mathcal{K}}^{2} \stackrel{P}{\rightarrow} \phi_{\mathcal{K}}^{2}
$$

Then $\widehat{J}_{n} \stackrel{D}{\rightarrow} \chi_{2}^{2}$ and $J_{n}^{\star} \stackrel{D}{\rightarrow} \chi_{2}^{2}$, where $\chi_{2}^{2}$ is a chi-square random variable with two degrees of freedom.

We now turn to the consistency of the estimators given by (2.12) and (2.13). For these results more restrictive assumptions on the spatial domain are required. Recall that $n^{\star}:=\min _{1 \leq i \leq d} n_{i}$ and $h^{\star}=\max _{1 \leq \ell \leq d} h_{\ell}$. 
TheOREm 2.2 Suppose (2.1), Assumptions 2.1-2.3 hold, and $h^{\star}=o\left(n^{\star 1 / 2}\right)$. Then relations (2.14) hold for the estimators $\hat{\phi}_{\mathcal{S}, \text { kern }}^{2}$ and $\hat{\phi}_{\mathcal{K}, \text { kern }}^{2}$ given by (2.12) and the estimators $\hat{\phi}_{\mathcal{S}, \text { pow }}^{2}$ and $\hat{\phi}_{\mathcal{K}, \text { pow }}^{2}$ given by $(2.13)$.

Estimation of the spatial long-run variance $\sigma^{2}$ given by (2.9) has been recently studied by Prause \& Steland (2018) who established consistency assuming $\varphi$-mixing with a suitable rate. If the errors $\varepsilon_{j}$ are normal, even for $d=1$, the moving average (2.2) is $\varphi$-mixing if only finitely many coefficients $a_{s}$ are not zero, see Ibragimov \& Linnik (1971) and Sidorov (2010). For this reason, we use a different, more direct, approach to prove Theorem 2.2.

We now turn to the consistency of the test. We begin with an assumption which is essentially Assumption 2.1, but without assuming normality.

Assumption 2.4 The $e_{i}$ are moving averages (2.2) with independent and identically distributed random variables $\varepsilon_{i}$, satisfying $E \varepsilon_{\ell}=0, E \varepsilon_{\ell}^{2}=1, E \varepsilon_{\ell}^{8}<\infty$, and the coefficients $a_{s}$ satisfying (2.3).

Under Assumption 2.4, we can establish limits in probability of $n_{\Gamma}^{-1 / 2} \mathcal{S}_{n}^{\star}$ and $n_{\Gamma}^{-1 / 2} \mathcal{K}_{n}^{\star}$, as stated in Theorem 2.3 below. Notice that under $H_{0}$ these limits are zero.

TheOREm 2.3 If (2.1) and Assumption 2.4 hold, then

$$
n_{\Gamma}^{-1 / 2} \mathcal{S}_{n}^{\star} \stackrel{P}{\rightarrow} E z_{0}^{3} \quad \text { and } \quad n_{\Gamma}^{-1 / 2} \mathcal{K}_{n}^{\star} \stackrel{P}{\rightarrow} E z_{0}^{4}-3,
$$

where $z_{0}$ is defined by (2.4). The limit of $n_{\Gamma}^{-1 / 2} \mathcal{K}_{n}$ is the same as the limit of $n_{\Gamma}^{-1 / 2} \mathcal{K}_{n}^{\star}$.

Next we establish bounds on magnitudes of the estimators of the long-run variances.

Theorem 2.4 Suppose (2.1) and Assumptions 2.3 and 2.4 hold, and $h^{\star}=o\left(n^{\star 1 / 2}\right)$. Then

$$
\hat{\phi}_{\mathcal{S}, \text { kern }}^{2}=O_{P}\left(h^{\star}\right), \quad \hat{\phi}_{\mathcal{K}, \text { kern }}^{2}=O_{P}\left(h^{\star}\right)
$$

and

$$
\hat{\phi}_{\mathcal{S}, \text { pow }}^{2}=O_{P}(1), \quad \hat{\phi}_{\mathcal{K}, \text { pow }}^{2}=O_{P}(1) .
$$

Using Theorems 2.3 and 2.4, we can prove the consistency of the test.

Corollary 2.2 If the conditions of Theorem 2.4 are satisfied and if $E z_{0}^{3} \neq 0$ and/or $E z_{0}^{4} \neq 3$, then $\widehat{J}_{n} \stackrel{P}{\rightarrow} \infty$ and $J_{n}^{\star} \stackrel{P}{\rightarrow} \infty$. 


\section{Finite sample performance and application to temperature data}

In Section 3.1, we explore the empirical size and power of several implementations of our test. In Section 3.2, we check if the spatial fields of sea surface temperature anomalies can be assumed to be Gaussian, and provide further insights into the behavior of the test.

\subsection{A simulation study}

In this section, we use Monte Carlo simulation to assess finite sample properties of the test derived in Section 2.1. We focus on the case of $d=2$, most commonly encountered in applications. Explicit formulas in this case are given in Section III of the Supplement. We consider data generating processes (DGPs) defined by three different spatial models specified below, and by several grid sizes. We use 5,000 independent replications, and record the count of rejections to calculate empirical size and power of the proposed test.

We generate realizations on a grid $\{1 \leq i, j \leq N\}$ of the following spatial models:

Spatial IID: $X_{i, j}=2+\sqrt{2} \xi_{i, j}$.

Spatial Moving-average (MA): $X_{i, j}=\xi_{i, j}+0.5 \xi_{i, j-1}$.

Spatial Autoregressive(AR): $X_{i, j}=0.5 X_{i-1, j-1}+\xi_{i, j}$.

Under $H_{0}, \xi_{i, j} \sim$ i.i.d. $\mathcal{N}(0,1)$. We consider two error distributions under $H_{A}$ : the $\xi_{i, j}$ are i.i.d. with either Student's $t$-distribution with $\nu$ degrees of freedom or with the skew-normal distribution. We set $\nu$ to values ranging from 5 to 20 . If $\nu \geq 30$, the univariate $t$-distribution is visually almost indistinguishable from the standard normal distribution, and its quantiles are almost equal to the standard normal quantiles. Unlike the $t$-distribution, the skew-normal distribution, treated in Azzalini (2014), has nonzero skewness. Further details and power tables are presented in Section IV of the Supplement.

Both the kernel and power estimators, defined in Section 2.2 (and Section III of the Supplement), need the specification of the kernel and the smoothing bandwidth. Three kernel functions are compared.

The truncated kernel (TR): $K_{T R}(t)=I\{|t| \leqslant 1\}$.

The Bartlett kernel (BT): $K_{B T}(t)=(1-|t|) I\{|t| \leqslant 1\}$.

The flat-top kernel (FT):

$$
K_{F T}(t)= \begin{cases}1, & 0 \leqslant t<0.5 \\ 2-|t|, & 0.5 \leqslant t<1 \\ 0, & 1 \leqslant t\end{cases}
$$


The bandwidth $h$ for these kernels is selected as

$$
h_{T R}=\left\lfloor 4(N / 100)^{1 / 5}\right\rfloor, \quad h_{B T}=\left\lfloor 4(N / 100)^{2 / 9}\right\rfloor, \quad h_{F T}=\left\lfloor 4(N / 100)^{1 / 5}\right\rfloor .
$$

The choice of the smoothing bandwidth has been well studied. For the truncated and Bartlett kernels, Newey \& West (1994) compared the performance of different plug-in methods, while Andrews (1991) proposed a data-driven bandwidth selection technique. Politis (2003) developed an adaptive bandwidth choice for the flat-top kernel. It turns out that these choices work well for our purpose. We thus follow Newey \& West (1994) to select the bandwidth for the truncated and Bartlett kernels. Our simulations showed that choosing the bandwidth of the flat-top kernel the same as for the truncated kernel produces stable and satisfactory results.

Empirical size Table 1 reports the empirical sizes, the percentages of rejections under $H_{0}$. As can be seen, the empirical sizes are close to the theoretical levels, even for small grid size, such as $N=100$. Comparing the results for the kernel estimator and the power estimator, it seems that there is no obvious pattern in the empirical sizes. The differences arising from the application of different kernels are small and do not exhibit any clear pattern either. We conclude that our test controls size very well, not matter which one of the six considered implementations is used.

Empirical power Tables 2 and 3 present the empirical power of the test by $5 \%$ significance level critical values with the spatial long run variance estimated, respectively, by the kernel estimator and the power estimator. As expected, the power increases with the grid size $N$. Comparing the results for the three DGPs, we find that the test has higher power under the spatial IID than the two models with spatial dependence. This could be expected, as both the MA and AR models lead to some averaging of the $\xi_{i, j}$, bringing the observations $X_{i, j}$ a bit closer to normality. There is no apparent difference when using different kernels under the spatial IID, but the Bartlett kernel occasionally has marginally higher power under the spatial MA and AR models. An important observation is that different results are produced by using the two spatial long run variance estimators. When the power estimator is used, the power is monotonously decreasing as the degrees of freedom $\nu$ of the $\xi_{i, j}$ grow. However, this pattern does not occur when the kernel estimator is employed. To be specific for the kernel estimator, the expected power behavior is observed for $\nu>8$, but not for $\nu \leqslant 8$. A reasonable explanation is that we use the $8^{\text {th }}$ moment of the Student's $t$-distribution when estimating $\phi_{\mathcal{K}}^{2}$. However, the $k$-th moment of a Student's $t$ random variable is well-defined only for $k<\nu$. For the power estimator, we only use the $4^{\text {th }}$ moment of observations in the spatial models generated by the Student's $t$ random variable. Comparing Tables 2 and 3, we can conclude that the power estimator has better power properties than the kernel estimator. Additionally, the power estimator is more broadly applicable as it requires fewer moments of the data. We note that the kernel 
estimator requires the existence of first eight moments of the distribution, but we are still interested in the impact on power of the kernel estimator if some of the first eight moments do not exist. Thus, we also report the power of the kernel estimator for $\nu=8,5$ in Table 2. The empirical power when the skew-normal distribution is employed, has similar behavior, except that we do not see nonmonotonic power for the kernel estimator; both estimators produces comparable results. The test is very powerful even for small departures of normality. Details are discussed in Section IV of the Supplement.

Broad conclusions Based on the simulations we performed, we recommend the implementation based on the power estimators (2.13) and any one of the three kernels listed in this section, with bandwidths given by (3.1).

We conclude this section by presenting in Table 4 the empirical size of the standard Jarque-Bera test. under independence, this standard test has correct size, as does our test, but under spatial dependence it has overinflated size, while our test controls the size very well. The distortion increases as the nominal size decreases, and exceeds $100 \%$ of the nominal size at the 1 percent level.

\subsection{Normality of Sea Surface Temperature anomalies}

Sea Surface Temperatures (SSTs) are closely linked with EI Niño/Southern Oscillation (ENSO) events, which are related to pattern changes in rainfall, wind speeds, ocean circulation, and general global weather patterns. The North Carolina Institute for Climate Studies (NCICS) provides monthly mean of daily Optimum Interpolation Sea Surface Temperature (OISST) analysis using Advanced Very High Resolution Radiometer (AVHRR) prepared for Observations for Model Intercomparisons Project by National Centers for Environmental Information (NCEI). The global SSTs are on a $1440 \times 720$ grid (in every 1/4 longitude degree and 1/4 latitude degree) observed daily for over 30 years, with missing pixels over land. The specific data we used was downloaded from the website https://esgf-node.1lnl.gov/search/obs4mips. In the dataset, there are 400 monthly observations in the period of September 1981 to December 2014. Figure 2 shows a snapshot of the SST data in the month of September 1981.

Denote the SST observations by $Y_{i, j}(t)$, where $t$ is a month, $i$ is longitude, and $j$ is latitude. These observations are available only for coordinates $i, j$ which correspond to sea, not to land. For any sufficiently large region, and any month $t$, the observations $Y_{i, j}(t)$ cannot be considered as a realization of a stationary spatial field because of spatial trends in water temperature due to latitude, ocean currents and the shape of neighboring land. We must therefore transform these data to consider them as a realization of a stationary random field whose normality can be tested. A transformation that is of primary interest, see e.g. NASA (2019), is defined as follows. Compute the long term averages

$$
A_{i, j}(T)=\frac{1}{T} \sum_{t=1}^{T} Y_{i, j}(t)
$$


Table 1: The empirical sizes of spatial normality test

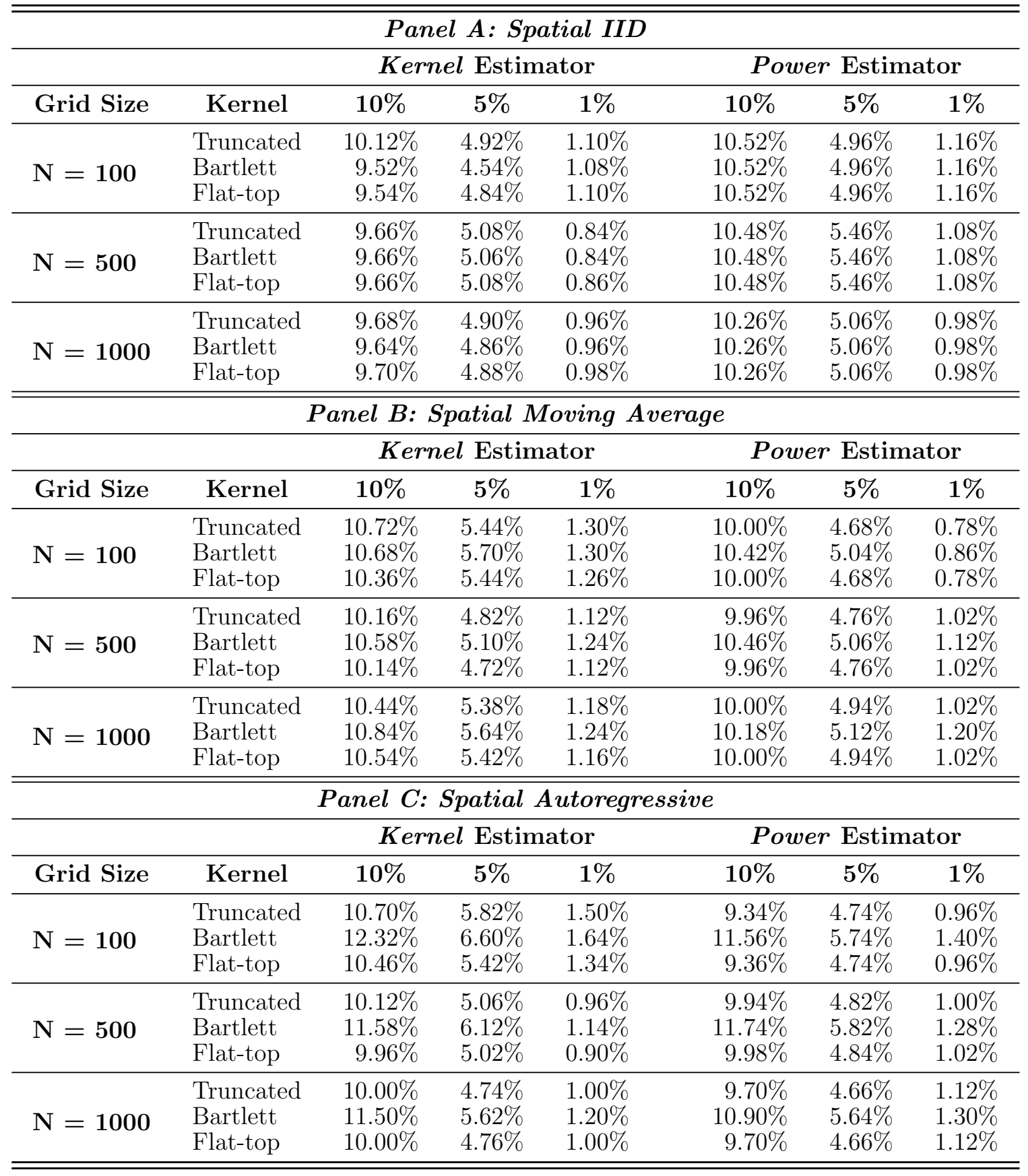


Table 2: Empirical power of the spatial normality test - kernel estimator

\begin{tabular}{|c|c|c|c|c|c|}
\hline \multicolumn{6}{|c|}{ Panel A: Spatial IID } \\
\hline Grid Size & Kernel & $\nu=20$ & $\nu=9$ & $\nu=8$ & $\nu=5$ \\
\hline \multirow{3}{*}{$\mathrm{N}=25$} & Truncated & $18.86 \%$ & $43.42 \%$ & $47.82 \%$ & $54.08 \%$ \\
\hline & Bartlett & $9.18 \%$ & $33.22 \%$ & $38.92 \%$ & $48.66 \%$ \\
\hline & Flat-top & $11.52 \%$ & $36.70 \%$ & $41.62 \%$ & $50.00 \%$ \\
\hline \multirow{3}{*}{$\mathrm{N}=50$} & Truncated & $54.86 \%$ & $90.14 \%$ & $88.12 \%$ & $76.64 \%$ \\
\hline & Bartlett & $53.52 \%$ & $90.20 \%$ & $88.10 \%$ & $75.94 \%$ \\
\hline & Flat-top & $54.36 \%$ & $90.22 \%$ & $88.18 \%$ & $76.14 \%$ \\
\hline \multirow{3}{*}{$\mathrm{N}=100$} & Truncated & $99.50 \%$ & $98.28 \%$ & $97.94 \%$ & $87.10 \%$ \\
\hline & Bartlett & $99.50 \%$ & $98.24 \%$ & $97.94 \%$ & $86.96 \%$ \\
\hline & Flat-top & $99.50 \%$ & $98.26 \%$ & $97.96 \%$ & $87.06 \%$ \\
\hline \multicolumn{6}{|c|}{ Panel B: Spatial Moving Average } \\
\hline Grid Size & Kernel & $\nu=20$ & $\nu=9$ & $\nu=8$ & $\nu=5$ \\
\hline \multirow{3}{*}{$\mathrm{N}=25$} & Truncated & $12.50 \%$ & $28.80 \%$ & $32.20 \%$ & $45.78 \%$ \\
\hline & Bartlett & $6.68 \%$ & $20.18 \%$ & $23.22 \%$ & $40.50 \%$ \\
\hline & Flat-top & $7.34 \%$ & $21.54 \%$ & $24.64 \%$ & $40.58 \%$ \\
\hline \multirow{3}{*}{$\mathrm{N}=50$} & Truncated & $28.34 \%$ & $82.56 \%$ & $84.18 \%$ & $73.96 \%$ \\
\hline & Bartlett & $27.62 \%$ & $83.32 \%$ & $84.78 \%$ & $74.82 \%$ \\
\hline & Flat-top & $26.86 \%$ & $81.94 \%$ & $83.78 \%$ & $73.58 \%$ \\
\hline \multirow{3}{*}{$\mathrm{N}=100$} & Truncated & $93.04 \%$ & $98.14 \%$ & $97.60 \%$ & $86.30 \%$ \\
\hline & Bartlett & $93.48 \%$ & $98.26 \%$ & $97.76 \%$ & $86.82 \%$ \\
\hline & Flat-top & $93.06 \%$ & $98.10 \%$ & $97.56 \%$ & $86.24 \%$ \\
\hline \multicolumn{6}{|c|}{ Panel C: Spatial Autoregressive } \\
\hline Grid Size & Kernel & $\nu=20$ & $\nu=9$ & $\nu=8$ & $\nu=5$ \\
\hline \multirow{3}{*}{$\mathrm{N}=25$} & Truncated & $11.90 \%$ & $22.80 \%$ & $26.44 \%$ & $41.64 \%$ \\
\hline & Bartlett & $7.44 \%$ & $17.50 \%$ & $20.58 \%$ & $38.70 \%$ \\
\hline & Flat-top & $6.68 \%$ & $16.38 \%$ & $18.94 \%$ & $35.84 \%$ \\
\hline \multirow{3}{*}{$\mathrm{N}=50$} & Truncated & $22.66 \%$ & $75.30 \%$ & $79.36 \%$ & $74.06 \%$ \\
\hline & Bartlett & $24.10 \%$ & $78.76 \%$ & $81.70 \%$ & $75.84 \%$ \\
\hline & Flat-top & $21.26 \%$ & $75.16 \%$ & $79.22 \%$ & $73.48 \%$ \\
\hline \multirow{3}{*}{$\mathrm{N}=100$} & Truncated & $84.44 \%$ & $97.70 \%$ & $97.20 \%$ & $86.32 \%$ \\
\hline & Bartlett & $86.60 \%$ & $98.10 \%$ & $97.50 \%$ & $87.52 \%$ \\
\hline & Flat-top & $84.42 \%$ & $97.76 \%$ & $97.18 \%$ & $86.16 \%$ \\
\hline
\end{tabular}


Table 3: Empirical power of the spatial normality test - power estimator

\begin{tabular}{|c|c|c|c|c|c|}
\hline \multicolumn{6}{|c|}{ Panel A: Spatial IID } \\
\hline Grid Size & Kernel & $\nu=20$ & $\nu=9$ & $\nu=8$ & $\nu=5$ \\
\hline \multirow{3}{*}{$\mathrm{N}=25$} & Truncated & $36.62 \%$ & $87.62 \%$ & $93.22 \%$ & $99.84 \%$ \\
\hline & Bartlett & $36.64 \%$ & $87.62 \%$ & $93.22 \%$ & $99.84 \%$ \\
\hline & Flat-top & $36.64 \%$ & $87.62 \%$ & $93.22 \%$ & $99.84 \%$ \\
\hline \multirow{3}{*}{$\mathrm{N}=50$} & Truncated & $82.44 \%$ & $100.00 \%$ & $100.00 \%$ & $100.00 \%$ \\
\hline & Bartlett & $82.44 \%$ & $100.00 \%$ & $100.00 \%$ & $100.00 \%$ \\
\hline & Flat-top & $82.44 \%$ & $100.00 \%$ & $100.00 \%$ & $100.00 \%$ \\
\hline \multirow{3}{*}{$\mathbf{N}=100$} & Truncated & $100.00 \%$ & $100.00 \%$ & $100.00 \%$ & $100.00 \%$ \\
\hline & Bartlett & $100.00 \%$ & $100.00 \%$ & $100.00 \%$ & $100.00 \%$ \\
\hline & Flat-top & $100.00 \%$ & $100.00 \%$ & $100.00 \%$ & $100.00 \%$ \\
\hline \multicolumn{6}{|c|}{ Panel B: Spatial Moving Average } \\
\hline Grid Size & Kernel & $\nu=20$ & $\nu=9$ & $\nu=8$ & $\nu=5$ \\
\hline \multirow{3}{*}{$\mathrm{N}=25$} & Truncated & $23.60 \%$ & $67.98 \%$ & $76.78 \%$ & $98.02 \%$ \\
\hline & Bartlett & $24.50 \%$ & $68.76 \%$ & $77.54 \%$ & $98.14 \%$ \\
\hline & Flat-top & $23.60 \%$ & $68.00 \%$ & $76.78 \%$ & $98.02 \%$ \\
\hline \multirow{3}{*}{$\mathrm{N}=50$} & Truncated & $56.30 \%$ & $99.36 \%$ & $99.86 \%$ & $100.00 \%$ \\
\hline & Bartlett & $57.02 \%$ & $99.42 \%$ & $99.86 \%$ & $100.00 \%$ \\
\hline & Flat-top & $56.30 \%$ & $99.36 \%$ & $99.86 \%$ & $100.00 \%$ \\
\hline \multirow{3}{*}{$\mathrm{N}=100$} & Truncated & $98.14 \%$ & $100.00 \%$ & $100.00 \%$ & $100.00 \%$ \\
\hline & Bartlett & $98.26 \%$ & $100.00 \%$ & $100.00 \%$ & $100.00 \%$ \\
\hline & Flat-top & $98.14 \%$ & $100.00 \%$ & $100.00 \%$ & $100.00 \%$ \\
\hline \multicolumn{6}{|c|}{ Panel C: Spatial Autoregressive } \\
\hline Grid Size & Kernel & $\nu=20$ & $\nu=9$ & $\nu=8$ & $\nu=5$ \\
\hline \multirow{3}{*}{$\mathrm{N}=25$} & Truncated & $19.66 \%$ & $59.84 \%$ & $68.24 \%$ & $96.00 \%$ \\
\hline & Bartlett & $22.60 \%$ & $63.08 \%$ & $71.02 \%$ & $96.56 \%$ \\
\hline & Flat-top & $19.82 \%$ & $60.16 \%$ & $68.48 \%$ & $96.04 \%$ \\
\hline \multirow{3}{*}{$\mathrm{N}=50$} & Truncated & $44.26 \%$ & $97.74 \%$ & $99.32 \%$ & $100.00 \%$ \\
\hline & Bartlett & $47.40 \%$ & $98.10 \%$ & $99.42 \%$ & $100.00 \%$ \\
\hline & Flat-top & $44.62 \%$ & $97.78 \%$ & $99.32 \%$ & $100.00 \%$ \\
\hline \multirow{3}{*}{$\mathrm{N}=100$} & Truncated & $93.34 \%$ & $100.00 \%$ & $100.00 \%$ & $100.00 \%$ \\
\hline & Bartlett & $94.10 \%$ & $100.00 \%$ & $100.00 \%$ & $100.00 \%$ \\
\hline & Flat-top & $93.34 \%$ & $100.00 \%$ & $100.00 \%$ & $100.00 \%$ \\
\hline
\end{tabular}


Table 4: The empirical sizes of Jarque-Bera test

\begin{tabular}{lrcc}
\hline \hline \multicolumn{4}{c}{ Panel A: Spatial IID } \\
\hline Grid Size & $\mathbf{1 0 \%}$ & $\mathbf{5 \%}$ & $\mathbf{1 \%}$ \\
\hline $\mathbf{N}=\mathbf{1 0 0}$ & $9.62 \%$ & $4.88 \%$ & $1.02 \%$ \\
$\mathbf{N}=\mathbf{5 0 0}$ & $10.48 \%$ & $4.70 \%$ & $0.98 \%$ \\
$\mathbf{N}=\mathbf{1 0 0 0}$ & $9.80 \%$ & $5.18 \%$ & $0.94 \%$ \\
\hline \hline \multicolumn{4}{c}{ Panel B: Spatial Moving Average } \\
\hline Grid Size & $\mathbf{1 0 \%}$ & $\mathbf{5 \%}$ & $\mathbf{1 \%}$ \\
\hline $\mathbf{N}=\mathbf{1 0 0}$ & $12.48 \%$ & $6.66 \%$ & $1.60 \%$ \\
$\mathbf{N}=\mathbf{5 0 0}$ & $11.96 \%$ & $6.74 \%$ & $1.76 \%$ \\
$\mathbf{N}=\mathbf{1 0 0 0}$ & $12.84 \%$ & $7.08 \%$ & $1.68 \%$ \\
\hline \hline \multicolumn{4}{c}{ Panel C: Spatial Autoregressive } \\
\hline Grid Size & $\mathbf{1 0 \%}$ & $\mathbf{5 \%}$ & $\mathbf{1 \%}$ \\
\hline $\mathbf{N}=\mathbf{1 0 0}$ & $15.20 \%$ & $8.68 \%$ & $2.36 \%$ \\
$\mathbf{N}=\mathbf{5 0 0}$ & $14.88 \%$ & $8.44 \%$ & $2.30 \%$ \\
$\mathbf{N}=\mathbf{1 0 0 0}$ & $14.80 \%$ & $8.10 \%$ & $2.22 \%$ \\
\hline \hline
\end{tabular}

where $T$ is number of the same calendar months in the sample period. For example, if $t$ corresponds to July, and we have $T=33$ Julys in the sample period. The monthly anomalies are defined as

$$
D_{i, j}(t)=Y_{i, j}(t)-A_{i, j}(T) .
$$

They are deviations in a given year from what is typical for a given month at location $(i, j)$. As quantified by French et al. (2019), among others, surface temperatures exhibit complex spatial trends in their variability. These are more pronounced over continents (temperatures over coastal regions are less variable that those in the interior), but one can expect a similar, though smaller, effect over bodies of water. We therefore also consider standardized anomalies defined by

$$
U_{i, j}(t)=\frac{Y_{i, j}(t)-A_{i, j}(T)}{S D_{i, j}(T)}
$$

where

$$
S D_{i, j}^{2}(T)=\frac{1}{T} \sum_{t=1}^{T}\left(Y_{i, j}(t)-A_{i, j}(T)\right)^{2} .
$$

As spatial domains, we selected four squared ocean regions with different characteristics. Region 1 (longitude $60^{\circ}$ to $90^{\circ}$, latitude $-30^{\circ}$ to $0^{\circ}$ ) lies in Indian Ocean and in the southern hemisphere. Region 2 (longitude $170^{\circ}$ to $200^{\circ}$, latitude $-15^{\circ}$ to $15^{\circ}$ ) is located in the Pacific Ocean and it is symmetric by the equator. Region 3 (longitude $210^{\circ}$ to $240^{\circ}$, 


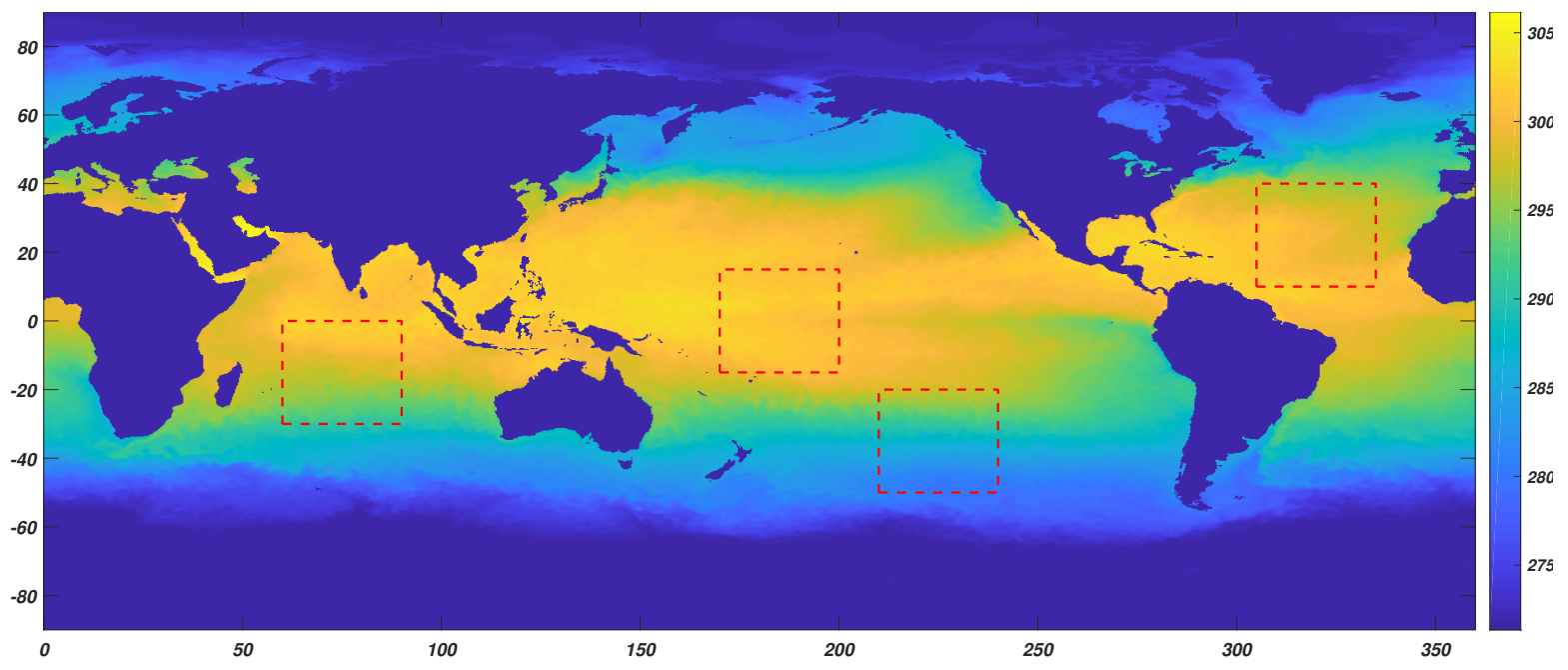

Figure 2: SST data snapshot in September 1981 and the four selected regions.

latitude $-50^{\circ}$ to $-20^{\circ}$ ) is also in the Pacific Ocean but it is in the southern hemisphere, away from the equator. The last region, Region 4 (longitude $305^{\circ}$ to $335^{\circ}$, latitude $10^{\circ}$ to $\left.40^{\circ}\right)$ is in the Northern Atlantic. The data over these regions are on a $120 \times 120$ grid, due to the fact that they all contains an area extending $30^{\circ}$ of longitude and $30^{\circ}$ of latitude. The four selected regions are highlighted in the Figure 2.

Conclusions from the application of the normality test We applied the implementations with both the kernel and the power estimator in order to see if the differences observed in Section 3.1 manifest themselves for the temperature data. It turns out that the kernel and the power estimators produce consistent results in the most cases, but not in all cases. We only reports results for the flat-top kernel as other kernels produce similar results.

The $\mathrm{P}$-values for July in all years of the sample period for $D_{i, j}(t)$ and $U_{i, j}(t)$ are shown in Tables 5 and 6, respectively. The $\mathrm{P}$-values for January, April, and October are provided in the Supplement. The most general observation is that normality of these spatial data cannot be assumed without further checks, so spatial statistics methods which rely on the assumption of Gaussianity must be used with caution. It might be best to use methods which do not assume Gaussianity. Comparing the results for the two versions of monthly anomalies, $D_{i, j}(t)$ and $U_{i, j}(t)$, they generally lead to the same conclusion, but $U_{i, j}(t)$ tends to produce more acceptances of normality, indicated by the $\mathrm{P}$-values greater than 5\%. This effect is however not very large. By looking at the results in different four regions, we see that Region 1, which is in the Indian Ocean and in the southern hemisphere, is the one with the highest number of normality in the July monthly anomalies for all years. In particular, the test on the second version of monthly anomalies, $U_{i, j}(t)$, using the power estimator for the long run variance suggests the normality in 21 out of 33 years. On the opposite side, Region 4, which is located in the Atlantic Ocean 
Table 5: P-values of the spatial normality test for $D_{i, j}(t)$ in July

\begin{tabular}{|c|c|c|c|c|c|c|c|c|}
\hline \multirow[b]{2}{*}{ Date } & \multicolumn{4}{|c|}{ Kernel Estimator } & \multicolumn{4}{|c|}{ Power Estimator } \\
\hline & Region 1 & Region 2 & Region 3 & Region 4 & Region 1 & Region 2 & Region 3 & Region 4 \\
\hline Jul-1982 & $3.3 \%$ & $0.0 \%$ & $36.7 \%$ & $0.0 \%$ & $9.0 \%$ & $0.0 \%$ & $34.5 \%$ & $0.0 \%$ \\
\hline Jul-1983 & $47.4 \%$ & $0.0 \%$ & $0.0 \%$ & $0.0 \%$ & $46.4 \%$ & $1.8 \%$ & $1.6 \%$ & $0.0 \%$ \\
\hline Jul-1984 & $0.0 \%$ & $0.1 \%$ & $0.0 \%$ & $0.0 \%$ & $0.0 \%$ & $1.4 \%$ & $2.5 \%$ & $0.0 \%$ \\
\hline Jul-1985 & $0.0 \%$ & $0.0 \%$ & $0.1 \%$ & $0.0 \%$ & $0.4 \%$ & $0.0 \%$ & $0.0 \%$ & $0.4 \%$ \\
\hline Jul-1986 & $4.7 \%$ & $0.0 \%$ & $84.8 \%$ & $0.0 \%$ & $5.4 \%$ & $0.2 \%$ & $85.8 \%$ & $0.0 \%$ \\
\hline Jul-1987 & $27.0 \%$ & $1.4 \%$ & $71.4 \%$ & $0.0 \%$ & $45.5 \%$ & $11.8 \%$ & $73.5 \%$ & $0.0 \%$ \\
\hline Jul-1988 & $11.9 \%$ & $0.0 \%$ & $1.5 \%$ & $3.6 \%$ & $8.0 \%$ & $0.0 \%$ & $3.2 \%$ & $16.0 \%$ \\
\hline Jul-1989 & $0.5 \%$ & $0.0 \%$ & $0.3 \%$ & $0.0 \%$ & $0.3 \%$ & $0.0 \%$ & $8.0 \%$ & $0.0 \%$ \\
\hline Jul-1990 & $0.1 \%$ & $0.0 \%$ & $0.0 \%$ & $0.0 \%$ & $0.0 \%$ & $0.0 \%$ & $1.5 \%$ & $0.0 \%$ \\
\hline Jul-1991 & $0.3 \%$ & $0.0 \%$ & $0.0 \%$ & $0.0 \%$ & $6.6 \%$ & $0.0 \%$ & $0.0 \%$ & $0.0 \%$ \\
\hline Jul-1992 & $0.0 \%$ & $50.1 \%$ & $0.0 \%$ & $0.0 \%$ & $0.0 \%$ & $57.6 \%$ & $0.1 \%$ & $0.0 \%$ \\
\hline Jul-1993 & $11.5 \%$ & $0.0 \%$ & $0.0 \%$ & $0.0 \%$ & $0.0 \%$ & $0.4 \%$ & $0.0 \%$ & $0.0 \%$ \\
\hline Jul-1994 & $4.1 \%$ & $0.0 \%$ & $0.1 \%$ & $0.0 \%$ & $20.3 \%$ & $0.0 \%$ & $1.4 \%$ & $0.0 \%$ \\
\hline Jul-1995 & $0.0 \%$ & $31.6 \%$ & $1.9 \%$ & $0.0 \%$ & $0.1 \%$ & $58.7 \%$ & $1.8 \%$ & $0.0 \%$ \\
\hline Jul-1996 & $11.0 \%$ & $0.0 \%$ & $0.0 \%$ & $0.0 \%$ & $25.4 \%$ & $0.0 \%$ & $1.0 \%$ & $0.1 \%$ \\
\hline Jul-1997 & $0.8 \%$ & $0.0 \%$ & $74.2 \%$ & $0.0 \%$ & $10.2 \%$ & $0.3 \%$ & $52.5 \%$ & $0.0 \%$ \\
\hline Jul-1998 & $1.0 \%$ & $0.0 \%$ & $1.9 \%$ & $15.6 \%$ & $5.3 \%$ & $0.0 \%$ & $4.0 \%$ & $33.7 \%$ \\
\hline Jul-1999 & $0.1 \%$ & $0.0 \%$ & $0.0 \%$ & $10.4 \%$ & $3.3 \%$ & $0.0 \%$ & $0.0 \%$ & $18.6 \%$ \\
\hline Jul-2000 & $0.5 \%$ & $0.0 \%$ & $0.0 \%$ & $0.0 \%$ & $3.4 \%$ & $0.0 \%$ & $0.0 \%$ & $0.0 \%$ \\
\hline Jul-2001 & $0.0 \%$ & $0.0 \%$ & $0.0 \%$ & $0.7 \%$ & $0.0 \%$ & $0.0 \%$ & $0.1 \%$ & $12.4 \%$ \\
\hline Jul-2002 & $7.8 \%$ & $0.0 \%$ & $0.0 \%$ & $0.0 \%$ & $34.0 \%$ & $0.0 \%$ & $0.0 \%$ & $0.0 \%$ \\
\hline Jul-2003 & $0.0 \%$ & $0.0 \%$ & $57.8 \%$ & $0.0 \%$ & $0.0 \%$ & $0.0 \%$ & $49.6 \%$ & $0.2 \%$ \\
\hline Jul-2004 & $2.9 \%$ & $18.5 \%$ & $51.9 \%$ & $0.0 \%$ & $2.8 \%$ & $26.5 \%$ & $66.6 \%$ & $0.1 \%$ \\
\hline Jul-2005 & $1.5 \%$ & $5.2 \%$ & $0.0 \%$ & $0.0 \%$ & $0.0 \%$ & $2.1 \%$ & $0.1 \%$ & $0.3 \%$ \\
\hline Jul-2006 & $0.2 \%$ & $23.8 \%$ & $54.0 \%$ & $0.0 \%$ & $0.0 \%$ & $40.3 \%$ & $57.0 \%$ & $0.0 \%$ \\
\hline Jul-2007 & $0.0 \%$ & $0.0 \%$ & $8.9 \%$ & $0.0 \%$ & $0.0 \%$ & $0.0 \%$ & $0.9 \%$ & $0.3 \%$ \\
\hline Jul-2008 & $37.0 \%$ & $0.0 \%$ & $0.1 \%$ & $0.0 \%$ & $41.9 \%$ & $0.0 \%$ & $0.0 \%$ & $0.0 \%$ \\
\hline Jul-2009 & $34.3 \%$ & $0.3 \%$ & $48.9 \%$ & $0.0 \%$ & $45.6 \%$ & $9.5 \%$ & $56.8 \%$ & $0.0 \%$ \\
\hline Jul-2010 & $4.1 \%$ & $9.7 \%$ & $0.0 \%$ & $0.0 \%$ & $13.2 \%$ & $21.5 \%$ & $0.3 \%$ & $0.0 \%$ \\
\hline Jul-2011 & $0.0 \%$ & $0.0 \%$ & $0.6 \%$ & $0.0 \%$ & $0.3 \%$ & $0.0 \%$ & $13.8 \%$ & $0.0 \%$ \\
\hline Jul-2012 & $0.0 \%$ & $0.0 \%$ & $0.0 \%$ & $0.1 \%$ & $0.3 \%$ & $0.0 \%$ & $1.0 \%$ & $2.5 \%$ \\
\hline Jul-2013 & $0.0 \%$ & $0.0 \%$ & $12.1 \%$ & $0.6 \%$ & $0.1 \%$ & $0.1 \%$ & $4.0 \%$ & $0.1 \%$ \\
\hline Jul-2014 & $0.2 \%$ & $0.0 \%$ & $53.7 \%$ & $0.8 \%$ & $0.3 \%$ & $0.0 \%$ & $49.4 \%$ & $2.0 \%$ \\
\hline
\end{tabular}


Table 6: P-values of the spatial normality test for $U_{i, j}(t)$ in July

\begin{tabular}{|c|c|c|c|c|c|c|c|c|}
\hline \multirow[b]{2}{*}{ Date } & \multicolumn{4}{|c|}{ Kernel Estimator } & \multicolumn{4}{|c|}{ Power Estimator } \\
\hline & Region 1 & Region 2 & Region 3 & Region 4 & Region 1 & Region 2 & Region 3 & Region 4 \\
\hline Jul-1982 & $11.3 \%$ & $0.0 \%$ & $0.0 \%$ & $0.0 \%$ & $40.3 \%$ & $0.0 \%$ & $0.0 \%$ & $0.0 \%$ \\
\hline Jul-1983 & $1.3 \%$ & $0.0 \%$ & $0.1 \%$ & $0.0 \%$ & $13.1 \%$ & $0.0 \%$ & $0.7 \%$ & $0.0 \%$ \\
\hline Jul-1984 & $0.0 \%$ & $6.8 \%$ & $0.0 \%$ & $0.0 \%$ & $0.2 \%$ & $29.3 \%$ & $0.1 \%$ & $0.0 \%$ \\
\hline Jul-1985 & $0.0 \%$ & $0.0 \%$ & $1.2 \%$ & $0.0 \%$ & $0.1 \%$ & $1.0 \%$ & $3.2 \%$ & $1.2 \%$ \\
\hline Jul-1986 & $0.4 \%$ & $0.0 \%$ & $0.3 \%$ & $0.0 \%$ & $0.1 \%$ & $0.0 \%$ & $6.3 \%$ & $0.0 \%$ \\
\hline Jul-1987 & $3.9 \%$ & $0.0 \%$ & $77.7 \%$ & $0.0 \%$ & $5.9 \%$ & $0.6 \%$ & $83.5 \%$ & $0.0 \%$ \\
\hline Jul-1988 & $56.3 \%$ & $0.0 \%$ & $5.8 \%$ & $0.0 \%$ & $70.9 \%$ & $0.1 \%$ & $15.8 \%$ & $0.0 \%$ \\
\hline Jul-1989 & $19.4 \%$ & $0.0 \%$ & $0.0 \%$ & $0.0 \%$ & $40.4 \%$ & $0.0 \%$ & $0.1 \%$ & $0.0 \%$ \\
\hline Jul-1990 & $70.5 \%$ & $0.1 \%$ & $1.6 \%$ & $0.1 \%$ & $82.5 \%$ & $0.3 \%$ & $7.7 \%$ & $0.4 \%$ \\
\hline Jul-1991 & $0.0 \%$ & $0.0 \%$ & $0.0 \%$ & $0.0 \%$ & $0.3 \%$ & $1.9 \%$ & $0.0 \%$ & $0.0 \%$ \\
\hline Jul-1992 & $8.0 \%$ & $0.1 \%$ & $0.0 \%$ & $0.0 \%$ & $12.1 \%$ & $0.1 \%$ & $0.0 \%$ & $0.3 \%$ \\
\hline Jul-1993 & $38.6 \%$ & $0.0 \%$ & $0.0 \%$ & $0.2 \%$ & $63.6 \%$ & $0.0 \%$ & $1.7 \%$ & $2.5 \%$ \\
\hline Jul-1994 & $17.9 \%$ & $0.0 \%$ & $0.0 \%$ & $0.0 \%$ & $43.1 \%$ & $0.0 \%$ & $1.5 \%$ & $0.0 \%$ \\
\hline Jul-1995 & $0.3 \%$ & $43.0 \%$ & $5.8 \%$ & $0.0 \%$ & $6.6 \%$ & $47.3 \%$ & $9.7 \%$ & $0.0 \%$ \\
\hline Jul-1996 & $0.0 \%$ & $1.4 \%$ & $1.4 \%$ & $0.0 \%$ & $2.3 \%$ & $14.4 \%$ & $3.4 \%$ & $0.1 \%$ \\
\hline Jul-1997 & $0.0 \%$ & $0.0 \%$ & $59.1 \%$ & $0.0 \%$ & $0.3 \%$ & $0.0 \%$ & $66.8 \%$ & $0.0 \%$ \\
\hline Jul-1998 & $0.2 \%$ & $0.1 \%$ & $0.1 \%$ & $0.0 \%$ & $6.9 \%$ & $4.4 \%$ & $1.9 \%$ & $0.0 \%$ \\
\hline Jul-1999 & $0.7 \%$ & $0.0 \%$ & $0.0 \%$ & $3.2 \%$ & $12.0 \%$ & $0.0 \%$ & $0.0 \%$ & $7.6 \%$ \\
\hline Jul-2000 & $0.0 \%$ & $0.0 \%$ & $0.0 \%$ & $0.0 \%$ & $1.4 \%$ & $0.0 \%$ & $0.0 \%$ & $0.0 \%$ \\
\hline Jul-2001 & $0.0 \%$ & $0.0 \%$ & $0.0 \%$ & $0.0 \%$ & $0.0 \%$ & $0.1 \%$ & $0.0 \%$ & $0.3 \%$ \\
\hline Jul-2002 & $1.2 \%$ & $0.0 \%$ & $0.0 \%$ & $0.0 \%$ & $16.1 \%$ & $1.1 \%$ & $0.0 \%$ & $0.0 \%$ \\
\hline Jul-2003 & $0.1 \%$ & $0.0 \%$ & $53.9 \%$ & $0.0 \%$ & $0.3 \%$ & $0.0 \%$ & $48.8 \%$ & $2.6 \%$ \\
\hline Jul-2004 & $11.0 \%$ & $0.0 \%$ & $0.0 \%$ & $0.0 \%$ & $21.1 \%$ & $0.0 \%$ & $0.8 \%$ & $0.1 \%$ \\
\hline Jul-2005 & $96.4 \%$ & $45.2 \%$ & $0.0 \%$ & $0.0 \%$ & $97.4 \%$ & $48.6 \%$ & $0.5 \%$ & $0.0 \%$ \\
\hline Jul-2006 & $3.1 \%$ & $46.0 \%$ & $0.0 \%$ & $0.1 \%$ & $2.4 \%$ & $60.6 \%$ & $2.5 \%$ & $0.8 \%$ \\
\hline Jul-2007 & $10.4 \%$ & $0.0 \%$ & $0.0 \%$ & $0.0 \%$ & $10.1 \%$ & $0.0 \%$ & $0.0 \%$ & $0.1 \%$ \\
\hline Jul-2008 & $0.4 \%$ & $0.0 \%$ & $13.6 \%$ & $4.7 \%$ & $5.4 \%$ & $0.0 \%$ & $0.3 \%$ & $6.7 \%$ \\
\hline Jul-2009 & $35.2 \%$ & $0.4 \%$ & $6.1 \%$ & $0.0 \%$ & $59.5 \%$ & $8.8 \%$ & $18.0 \%$ & $0.0 \%$ \\
\hline Jul-2010 & $0.1 \%$ & $0.0 \%$ & $30.9 \%$ & $0.0 \%$ & $4.1 \%$ & $0.0 \%$ & $54.4 \%$ & $0.0 \%$ \\
\hline Jul-2011 & $3.9 \%$ & $0.0 \%$ & $0.0 \%$ & $0.0 \%$ & $19.8 \%$ & $0.0 \%$ & $0.0 \%$ & $0.0 \%$ \\
\hline Jul-2012 & $0.0 \%$ & $0.0 \%$ & $0.0 \%$ & $0.0 \%$ & $0.2 \%$ & $0.0 \%$ & $0.6 \%$ & $1.2 \%$ \\
\hline Jul-2013 & $13.3 \%$ & $0.0 \%$ & $34.2 \%$ & $76.3 \%$ & $25.5 \%$ & $0.0 \%$ & $44.9 \%$ & $81.3 \%$ \\
\hline Jul-2014 & $9.1 \%$ & $0.0 \%$ & $0.0 \%$ & $0.0 \%$ & $28.2 \%$ & $0.0 \%$ & $1.5 \%$ & $1.2 \%$ \\
\hline
\end{tabular}


and in the northern hemisphere, has the lowest number of acceptances of normality of the July monthly anomalies. Specifically, $U_{i, j}(t)$ with the power estimator only suggests the normality in 3 out of 33 years. These conclusions also hold for other months.

\section{References}

Anderes, E. B. \& Stein, M. L. (2008), Estimating deformations of isotropic Gaussian random fields on the plane, The Annals of Statistics 36, 719-741.

Anderson, T. W. \& Darling, D. A. (1954), A test of goodness-of-fit, Journal of the American Statistical Association 49, 765-769.

Andrews, D. W. K. (1991), Heteroskedasticity and autocorrelation consistent covariance matrix estimation, Econometrica 59, 817-858.

Azzalini, A. (2014), The Skew-Normal and Related Families, IMS.

Banerjee, S., Carlin, B. \& Gelfand, A. (2014), Hierarchical Modeling and Analysis for Spatial Data, CRC Press.

Billingsley, P. (1968), Convergence of Probability Measures, Wiley, New York.

Chang, M.-C., Cheng, S.-W. \& Cheng, C.-S. (2019), Signal aliasing in Gaussian random fields for experiments with quantitative factors, The Annals of Statistics 47, 909-935.

Cressie, N. (1993), Statistics for Spatial Data, Wiley.

Cressie, N. \& Wikle, C. (2011), Statistics for Spatio-Temporal Data, Wiley.

D’Agostino, R. B., Belanger, A. \& D'Agostino Jr., R. B. (1990), A suggestion for using powerful and informative tests of normality, The American Statistician 44, 316-321.

Doornik, J. A. \& Hansen, H. (2008), An omnibus test for univariate and multivariate normality, Oxford Bulletin of Economics and Statistics 70, 927-939.

El Machkouri, M., Volný, D. \& Wu, W. B. (2013), A central limit theorem for stationary random fields, Stochastic Processes and their Applications 123, 1-14.

Fouedjio, F., Desassis, N. \& Romary, T. (2015), Estimation of space deformation model for non-stationary random functions, Spatial Statistics 13, 45-61.

French, J., Kokoszka, P., Stoev, S. \& Hall, L. (2019), Quantifying the risk of heat waves using extreme value theory and spatio-temporal functional data, Computational Statistics and Data Analysis 131, 176-193.

Gelfand, A. E., Diggle, P. J., Fuentes, M. \& Guttorp, P., eds (2010), Handbook of Spatial Statistics, CRC Press. 
Gelfand, A. \& Schliep, E. (2016), Spatial statistics and Gaussian processes: A beautiful marriage, Spatial statistics 18, 86-104.

Guhaniyogi, R. \& Banerjee, S. (2018), Meta-kriging: Scalable Bayesian modeling and inference for massive spatial datasets, Technometrics 60, 430-444.

Henze, N. \& Zirkler, B. (1990), A class of invariant consistent tests for multivariate normality, Communications in Statistics - Theory and Methods 19, 3595-3617.

Ibragimov, I. A. \& Linnik, Y. V. (1971), Independent and Stationary Sequences of Random Variables, Wolters-Nordhoff,.

Jarque, C. M. \& Bera, A. K. (1980), Efficient tests for normality, homoskedasticity and serial independence of regression residuals, Economic Letters 6, 255-259.

Jarque, C. M. \& Bera, A. K. (1987), A test of normality of observations and regression residual, International Statistical Review 55, 163-172.

Katzfuss, M. (2017), A multi-resolution approximation for massive spatial datasets, Journal of the American Statistical Association 112, 201-214.

Lahiri, S. \& Robinson, P. (2016), Central limit theorems for long range dependent spatial linear processes, Bernoulli 22, 345-375.

Mardia, K. V. (1970), Measures of multivariate skewness and kurtosis with applications, Biometrika 57, 519-530.

Mardia, K. V. (1974), Applications of some measures of multivariate skewness and kurtosis in testing normality and robustness studies, Sankhya (B) 36, 115-128.

NASA (2019), 'Sea Surface Temperature Anomaly Global Maps', NASA Earth Observatory, earthobservatory.nasa.gov/global-maps/AMSRE_SSTAn_M. Accessed April 2019.

Newey, W. \& West, K. (1994), Automatic lag selection in covariance matrix estimation, The Review of Economic Studies 61, 631-653.

Nychka, D., Bandyopadhyay, S., Hammerling, D., Lindgren, F. \& Sain, S. (2015), A multiresolution Gaussian process model for the analysis of large spatial datasets, Journal of Computational and Graphical Statistics 24, 579-599.

Paciorek, C., Lipshitz, B., Zhuo, W., Kaufman, C. \& Thomas, R. (2015), Parallelizing Gaussian process calculations in R, Journal of Statistical Software 63, 1-23.

Politis, D. (2003), Adaptive bandwidth choice, Journal of Nonparametric Statistics 15, 517-533.

Prause, A. \& Steland, A. (2018), Estimation of the asymptotic variance of univariate and multivariate random fields and statistical inference, Electronic Journal of Statistics 12, 890-940. 
Royston, J. P. (1982), An extension of shapiro and wilk's w test for normality to large samples, Journal of the Royal Statistical Society. Series C (Applied Statistics) 31(2), 115-124.

Royston, J. P. (1983), Some techniques for assessing multivarate normality based on the ShapiroWilk W, Journal of the Royal Statistical Society (C) 32(2), 121-133.

Royston, J. P. (1992), Approximating the Shapiro-Wilk W-test for non-normality, Statistics and Computing 2(3), 117-119.

Sampson, P. D. \& Guttorp, P. (1992), Nonparametric estimation of nonstationary spatial covariance structure, Journal of the American Statistical Association 87, 108-119.

Schabenberger, O. \& Gotway, C. A. (2005), Statistical Methods for Spatial Data Analysis, Chapman \& Hall/CRC.

Schmidt, A. M. \& O’Hogan, A. (2003), Bayesian inference for non-stationary spatial covariance structure via spatial deformations, Journal of the Royal Statistical Society: Series B 65, 743758.

Scholz, F. W. \& Stephens, M. A. (1997), K-sample Anderson-Darling tests, Journal of the American Statistical Association 82, 918-924.

Shapiro, S. S. \& Wilk, M. B. (1965), An analysis of variance test for normality (complete samples), Biometrika 52, 591-611.

Sidorov, D. I. (2010), On mixing conditions for sequences of moving averages, Theory of Probability and Its Applications 54, 339-347.

Stein, M. L. (1999), Interpolation of Spatial Data: Some Theory for Krigging, Springer.

Stephens, M. A. (1974), EDF statistics for goodness of fit and some comparisons", Journal of the American Statistical Association 69, 730-737.

Stroud, J., Stein, M. \& Lysen, S. (2017), Bayesian and maximum likelihood estimation for Gaussian processes on an incomplete lattice, Journal of Computational and Graphical Statistics $26,108-120$. 


\section{Supplement}

\section{Analysis of the Mercer and Hall wheat-yield data}

We present in this section a more detailed analysis of the Mercer and Hall wheat-yield data shown in Figure 1 of the main paper. The data were collected from wheat uniformity trials carried out at Rothamsted Experimental Station in 1910. The yield data are on 20 $\times 25$ grid with each slot in a size of approximately 3.30 meter (east-west) $\times 2.5$ meter (north-south), giving the total area of roughly one acre.

It is argued in Cressie (1993) that no transformation of these data is needed to ensure stationarity. Our test is carried out based on the original scale of pounds (of grain). To reveal the impact of different implementations of our test, we employ both the kernel estimators and the power estimators with three specification of the kernel. Table 7 presents the test statistics $J_{n}^{*}$ and their $\mathrm{P}$-values. Depending on the implementation, the $\mathrm{P}$-values are between $16 \%$ and $52 \%$. Our significance test shows that these data can be assumed to be a realization of a Gaussian process.

Table 7: Test Result of the Wheat-Yield Data

\begin{tabular}{llcc}
\hline & & $J_{n}^{*}$ & P-value \\
\hline \multirow{3}{*}{ Kernel Estimator } & Truncated & 1.93 & $38.17 \%$ \\
& Bartlett & 3.61 & $16.45 \%$ \\
& Flat-top & 3.70 & $15.75 \%$ \\
\hline \multirow{3}{*}{ Power Estimator } & Truncated & 1.24 & $53.90 \%$ \\
& Bartlett & 1.43 & $48.99 \%$ \\
& Flat-top & 1.31 & $51.84 \%$ \\
\hline
\end{tabular}

\section{Proofs of the results of Section 2}

\section{II.1 Proof of Theorem 2.1}

The absolute convergence of series (2.7) and (2.8) defining, respectively, $\phi_{\mathcal{S}}^{2}$ and $\phi_{\mathcal{K}}^{2}$ follows from Lemma II.1. This lemma holds under Assumption 2.4, which is more general than Assumption 2.1. The remaining claims of Theorem 2.1 follow immediately from Lemmas II.3, II.4, II.5 and part 2) of Lemma II.2. The main work is done in the proofs of Lemmas II.3, II.4 and II.5, which show that approximating the spatial array elements $x_{i}=x_{i, n}$ by the unobservable $z_{i}$ has an asymptotically negligible effect. 
LEMMA II.1 If Assumption 2.4 holds, then for all integers $1 \leq p, q \leq 8$,

$$
\sum_{i \in \mathbb{Z}^{d}}\left|E\left[z_{0}^{p} z_{i}^{q}\right]\right|<\infty
$$

ProOF: Without loss of generality, we can assume that $z_{i}=\sum_{s} a_{s} \varepsilon_{i-s}$. We will display the proof for $p=2$ and $q=1$, the idea is the same for any $1 \leq p, q \leq 8$, the number of indexes is larger and formulas more cumbersome. Observe that

$$
\begin{aligned}
E\left[z_{0}^{2} z_{i}\right] & =E\left[\left(\sum_{t} a_{t} \varepsilon_{-t}\right)^{2}\left(\sum_{s} a_{s} \varepsilon_{i-s}\right)\right] \\
& =E\left[\left(\sum_{t} a_{t} \varepsilon_{-t}\right)\left(\sum_{u} a_{u} \varepsilon_{-u}\right)\left(\sum_{s} a_{s} \varepsilon_{i-s}\right)\right] .
\end{aligned}
$$

Since $E \varepsilon_{k}=0$, using their independence and the fact that the $a_{k}$ are absolutely summable, we see that $E\left[z_{0}^{2} z_{i}\right]=\sum_{s} a_{s-i}^{2} a_{s} E \varepsilon_{0}^{3}$. It remains to notice that

$$
\sum_{i}\left|\sum_{s} a_{s-i}^{2} a_{s}\right| \leq \sum_{s}\left|a_{s}\right| \sum_{i} a_{s-i}^{2}=\sum_{s}\left|a_{s}\right| \sum_{t} a_{t}^{2}<\infty .
$$

LEMma II.2 1) If condition (2.1) and Assumption 2.4 hold, then the following sums are of the order $O_{P}\left(n_{\Gamma}^{1 / 2}\right)$ :

$$
\sum_{i \in \Gamma_{n}} z_{i}, \quad \sum_{i \in \Gamma_{n}}\left(z_{i}^{2}-1\right), \quad \sum_{i \in \Gamma_{n}} z_{i}^{3}, \quad \sum_{i \in \Gamma_{n}}\left(z_{i}^{4}-3\right) .
$$

2) If condition (2.1) and Assumption 2.1 hold, then the following convergence holds:

$$
\frac{1}{n_{\Gamma}^{1 / 2}} \sum_{i \in \Gamma_{n}}\left[\begin{array}{c}
z_{i}^{3}-3 z_{i} \\
z_{i}^{4}-6 z_{i}^{2}+6
\end{array}\right] \stackrel{D}{\rightarrow} N_{2}\left(\left[\begin{array}{l}
0 \\
0
\end{array}\right],\left[\begin{array}{cc}
\phi_{\mathcal{S}}^{2} & 0 \\
0 & \phi_{\mathcal{K}}^{2}
\end{array}\right]\right) .
$$

Proof: Claim 1) is an immediate consequence of Proposition 2 of El Machkouri et al. (2013). Claim 2) follows from Proposition 2 of El Machkouri et al. (2013) and the CramérWold device, see e.g. Billingsley (1968), p. 49, as we now explain.

Let $\lambda_{1}$ and $\lambda_{2}$ be two constants. Proposition 2 of El Machkouri et al. (2013) yields that

$$
\frac{1}{n_{\Gamma}^{1 / 2}} \sum_{i \in \Gamma_{n}}\left[\lambda_{1}\left(z_{i}^{3}-3 z_{i}\right)+\lambda_{2}\left(z_{i}^{4}-6 z_{i}^{2}+3\right)\right] \stackrel{D}{\rightarrow} N\left(0, \tau^{2}\right),
$$

where

$$
\tau^{2}=\sum_{i \in \mathbb{Z}^{d}} E\left\{E\left[\lambda_{1}\left(z_{0}^{3}-3 z_{0}\right)+\lambda_{2}\left(z_{0}^{4}-6 z_{0}^{2}+3\right)\right]\left[\lambda_{1}\left(z_{i}^{3}-3 z_{i}\right)+\lambda_{2}\left(z_{i}^{4}-6 z_{i}^{2}+3\right)\right]\right\} .
$$


Using Assumption 2.1, one can check directly that for all $i \in \mathbb{Z}^{d}$,

$$
E\left[\left(z_{0}^{3}-3 z_{0}\right)\left(z_{i}^{4}-6 z_{i}^{2}+3\right)\right]=0, \quad E\left[\left(z_{0}^{4}-6 z_{0}^{2}+3\right)\left(z_{i}^{3}-3 z_{i}\right)\right]=0 .
$$

Hence $\tau^{2}=\lambda_{1}^{2} \phi_{\mathcal{S}}^{2}+\lambda_{2}^{2} \phi_{\mathcal{K}}^{2}$, completing the verification of claim 2).

LEMma II.3 Under the assumptions of Theorem 2.1,

$$
\mathcal{S}_{n}=\frac{1}{n_{\Gamma}^{1 / 2}} \sum_{i \in \Gamma_{n}}\left(z_{i}^{3}-3 z_{i}\right)+o_{P}(1) .
$$

ProOF: Recall that $\mathcal{S}_{n}=n_{\Gamma}^{-1 / 2} \sum_{i \in \Gamma_{n}} x_{i}^{3}$. Due to the scaling in the definition of the $x_{i}$, we can assume without loss of generality that $\mu=0$ and $\sigma^{2}=1$. Set.

$$
\bar{z}=\frac{1}{n_{\Gamma}} \sum_{i \in \Gamma_{n}} z_{i}, \quad s_{z}^{2}=\frac{1}{n_{\Gamma}} \sum_{i \in \Gamma_{n}}\left(z_{i}-\bar{z}\right)^{2} .
$$

Under the assumption $\mu=0$ and $\sigma^{2}=1, \bar{z}=\bar{x}$ and $s_{z}^{2}=S^{2}$. Elementary algebra gives

$$
\frac{1}{n_{\Gamma}^{1 / 2}} \sum_{i \in \Gamma_{n}} x_{i}^{3}=\frac{1}{n_{\Gamma}^{1 / 2} s_{z}^{3}}\left\{\sum_{i \in \Gamma_{n}} z_{i}^{3}-3 n_{\Gamma} \bar{z}\right\}-\frac{3}{n_{\Gamma}^{1 / 2} s_{z}^{3}} \bar{z} \sum_{i \in \Gamma_{n}}\left(z_{i}^{2}-1\right)+\frac{2}{n_{\Gamma}^{1 / 2} s_{z}^{3}} n_{\Gamma}^{3 / 2} \bar{z}^{3} .
$$

Next we verify that

$$
s_{z}^{2} \stackrel{P}{\rightarrow} 1 .
$$

This follows from part 1) of Lemma II.2 because

$$
s_{z}^{2}=\frac{1}{n_{\Gamma}} \sum_{i \in \Gamma_{n}}\left(z_{i}-\bar{z}\right)^{2}=1+\frac{1}{n_{\Gamma}} \sum_{i \in \Gamma_{n}}\left(z_{i}^{2}-1\right)-\left(\frac{1}{n_{\Gamma}} \sum_{i \in \Gamma_{n}} z_{i}\right)^{2} .
$$

Using Lemma II.2 again, we thus obtain, for the last term,

$$
\frac{1}{n_{\Gamma}^{1 / 2} s_{z}^{3}} n_{\Gamma}^{3 / 2} \bar{z}^{3}=\frac{1}{s_{z}^{3}} n_{\Gamma}\left(\frac{1}{n_{\Gamma}} \sum_{i \in \Gamma_{n}} z_{i}\right)^{3}=n_{\Gamma} n_{\Gamma}^{-3 / 2} O_{P}(1)=o_{P}(1) .
$$

Similarly, for the second term,

$$
\frac{3}{n_{\Gamma}^{1 / 2} s_{z}^{3}} \bar{z} \sum_{i \in \Gamma_{n}}\left(z_{i}^{2}-1\right)=O_{P}\left(n_{\Gamma}^{-1 / 2}\right)=o_{P}(1) .
$$

For the first term, setting $D_{n}=n_{\Gamma}^{-1 / 2} \sum_{i \in \Gamma_{n}}\left(z_{i}^{3}-3 z_{i}\right)$, we get

$$
\frac{1}{n_{\Gamma}^{1 / 2} s_{z}^{3}}\left\{\sum_{i \in \Gamma_{n}} z_{i}^{3}-3 n_{\Gamma} \bar{z}\right\}=\frac{D_{n}}{s_{z}^{3}}=D_{n}+D_{n}\left(\frac{1}{s_{z}^{3}}-1\right) .
$$

By Lemma II.2, $D_{n}=O_{P}(1)$, so the second term is $o_{P}(1)$, and the claim follows.

LEMma II.4 Under the assumptions of Theorem 2.1,

$$
\mathcal{K}_{n}=\frac{1}{n_{\Gamma}^{1 / 2}} \sum_{i \in \Gamma_{n}}\left(z_{i}^{4}-6 z_{i}^{2}+3\right)+o_{P}(1) .
$$


Proof: It is easy to show that

$$
\begin{aligned}
s_{z}^{4}-1 & =\frac{1}{n_{\Gamma}^{2}}\left\{\left(\sum_{i \in \Gamma_{n}}\left(z_{i}-\bar{z}\right)^{2}\right)^{2}-n_{\Gamma}^{2}\right\} \\
& =\frac{1}{n_{\Gamma}^{2}}\left\{\left(\sum_{i \in \Gamma_{n}}\left(z_{i}-\bar{z}\right)^{2}-n_{\Gamma}\right)\left(\sum_{i \in \Gamma_{n}}\left(z_{i}-\bar{z}\right)^{2}+n_{\Gamma}\right)\right\} .
\end{aligned}
$$

By (II.4),

$$
\sum_{i \in \Gamma_{n}}\left(z_{i}-\bar{z}\right)^{2}=n_{\Gamma}+\sum_{i \in \Gamma_{n}}\left(z_{i}^{2}-1\right)-\left(n_{\Gamma}^{-1 / 2} \sum_{i \in \Gamma_{n}} z_{i}\right)^{2}
$$

Hence

$$
\begin{aligned}
s_{z}^{4}-1 & =\frac{1}{n_{\Gamma}^{2}}\left(\sum_{i \in \Gamma_{n}}\left(z_{i}^{2}-1\right)-\left(n_{\Gamma}^{-1 / 2} \sum_{i \in \Gamma_{n}} z_{i}\right)^{2}\right)\left(2 n_{\Gamma}+O_{P}\left(n_{\Gamma}^{1 / 2}\right)\right) \\
& =\frac{2}{n_{\Gamma}} \sum_{i \in \Gamma_{n}}\left(z_{i}^{2}-1\right)+n_{\Gamma}^{-1 / 2} \sum_{i \in \Gamma_{n}}\left(z_{i}^{2}-1\right) O_{P}\left(n_{\Gamma}^{-1}\right)+O_{P}\left(n_{\Gamma}^{-1}\right) \\
& =\frac{2}{n_{\Gamma}} \sum_{i \in \Gamma_{n}}\left(z_{i}^{2}-1\right)+O_{P}\left(n_{\Gamma}^{-1}\right) .
\end{aligned}
$$

Thus, we get

$$
\begin{aligned}
& \frac{1}{n_{\Gamma}^{1 / 2}} \sum_{i \in \Gamma_{n}}\left(x_{i}^{4}-3\right)=\frac{1}{n_{\Gamma}^{1 / 2} s_{z}^{4}} \sum_{i \in \Gamma_{n}}\left[\left(z_{i}-\bar{z}\right)^{4}-3 s_{z}^{4}\right] \\
& =\frac{1}{n_{\Gamma}^{1 / 2} s_{z}^{4}}\left\{\sum_{i \in \Gamma_{n}} z_{i}^{4}-4 \bar{z} \sum_{i \in \Gamma_{n}} z_{i}^{3}+6 \bar{z}^{2} \sum_{i \in \Gamma_{n}} z_{i}^{2}-4 \bar{z}^{3} \sum_{i \in \Gamma_{n}} z_{i}-3 n_{\Gamma} s_{z}^{4}\right\} \\
& =\frac{1}{n_{\Gamma}^{1 / 2} s_{z}^{4}}\left\{\sum_{i \in \Gamma_{n}} z_{i}^{4}-3 n_{\Gamma}-6 \sum_{i \in \Gamma_{n}}\left(z_{i}^{2}-1\right)+O_{P}(1)\right\} \\
& =\frac{1}{n_{\Gamma}^{1 / 2} s_{z}^{4}} \sum_{i \in \Gamma_{n}}\left(z_{i}^{4}-6 z_{i}^{2}+3\right)+O_{P}\left(n_{\Gamma}^{-1 / 2}\right) \\
& =\frac{1}{n_{\Gamma}^{1 / 2}} \sum_{i \in \Gamma_{n}}\left(z_{i}^{4}-6 z_{i}^{2}+3\right)+O_{P}\left(n_{\Gamma}^{-1 / 2}\right) \\
& +\left(\frac{1}{s_{z}^{4}}-1\right)\left\{\frac{1}{n_{\Gamma}^{1 / 2}} \sum_{i \in \Gamma_{n}}\left(z_{i}^{4}-3\right)-\frac{3}{n_{\Gamma}^{1 / 2}} \sum_{i \in \Gamma_{n}}\left(z_{i}^{2}-1\right)\right\} .
\end{aligned}
$$

By (II.3) and part 1) of Lemma II.2, the last term is $o_{P}(1)$, completing the proof of Lemma II.4. 
LEMMA II.5 Under the assumptions of Theorem 2.1,

$$
\mathcal{K}_{n}^{\star}=\frac{1}{n_{\Gamma}^{1 / 2}} \sum_{i \in \Gamma_{n}}\left(z_{i}^{4}-6 z_{i}^{2}+3\right)+o_{P}(1) .
$$

PRoOF: Recall that

$$
\mathcal{K}_{n}^{\star}=\frac{1}{n_{\Gamma}^{1 / 2}} \sum_{i \in \Gamma_{n}}\left(x_{i}^{4}-6 x_{i}^{2}+3\right), \quad \mathcal{K}_{n}=\frac{1}{n_{\Gamma}^{1 / 2}} \sum_{i \in \Gamma_{n}}\left(x_{i}^{4}-3\right) .
$$

The leading terms in Lemmas II.4 and II.5 are the same, so it is enough to verify that $n_{\Gamma}^{-1 / 2} \sum_{i \in \Gamma_{n}}\left(x_{i}^{2}-1\right)=o_{P}(1)$. Assuming again $\mu=0, \sigma^{2}=1$, we must thus show

$$
\frac{1}{n_{\Gamma}^{1 / 2} s_{z}^{2}} \sum_{i \in \Gamma_{n}}\left[\left(z_{i}-\bar{z}\right)^{2}-s_{z}^{2}\right]=o_{P}(1)
$$

Expanding the square and noticing that $n_{\Gamma}^{1 / 2} \bar{z}^{2}=O_{P}\left(n_{\Gamma}^{-1 / 2}\right)$, this reduces to showing that

$$
\frac{1}{n_{\Gamma}^{1 / 2} s_{z}^{2}} \sum_{i \in \Gamma_{n}}\left[z_{i}^{2}-s_{z}^{2}\right]=o_{P}(1)
$$

A direct calculation shows that $\sum_{i \in \Gamma_{n}}\left[z_{i}^{2}-s_{z}^{2}\right]=n \bar{z}^{2}=O_{P}(1)$, completing the proof.

\section{II.2 Proofs of Theorem 2.2}

The general plan of the proof is to first establish the convergence of the estimators based on the unobservable $z_{i}$ given by (2.4) and then show that replacing them by the $x_{i}$ given by (2.5) is asymptotically negligible. Lemmas II.6-II.8 establish results needed to prove the consistency of the kernel estimators (2.12). In Lemmas II.6 and II.7, $\Gamma$ is any finite subset of $\mathbb{Z}^{d}$.

LEMmA II.6 Under Assumption 2.1, for any $k \in \mathbb{Z}^{d}$, and any integers $r \geq 1$ and $q \geq 1$,

$$
E\left|\sum_{i \in \Gamma}\left(e_{i}^{r} e_{i+k}^{q}-E\left[e_{0}^{r} e_{k}^{q}\right]\right)\right| \leq C(r, q)|\Gamma|^{1 / 2} .
$$

PRoOF: We will work with the coupled random variables $e_{i}^{*}$ defined in Section 2 of El Machkouri et al. (2013). In the setting of Assumption 2.1, these are defined by $e_{i}^{*}=$ $\sum_{s \in \mathbb{Z}^{d}} a_{s} \varepsilon_{i-s}^{*}$, where $\varepsilon_{j}^{*}=\varepsilon_{j}$ for $j \neq 0$ and $\varepsilon_{0}^{*}=\varepsilon_{0}^{\prime}$, and where $\left\{\varepsilon_{j}^{\prime}\right\}$ is and independent copy of the field $\left\{\varepsilon_{j}^{\prime}\right\}$. This means that in the moving average (2.2), $\varepsilon_{i-s}$ is replaced by $\varepsilon_{i-s}^{\prime}$. The idea of the proof is to show that the field $\left\{e_{i}^{r} e_{i+k}^{q}-E\left[e_{0}^{r} e_{k}^{q}\right]\right\}$ satisfies the assumptions of Proposition 1 of El Machkouri et al. (2013) and to use this proposition. 
By the triangle inequality,

$$
\begin{aligned}
\left|e_{i}^{r} e_{i+k}^{q}-e_{i}^{* r} e_{i+k}^{* q}\right| \leq & \left|e_{i}^{r}\left(e_{i+k}^{q}-e_{i+k}^{* q}\right)\right|+\left|e_{i+k}^{* q}\left(e_{i}^{r}-e_{i}^{* r}\right)\right| \\
\leq & \left|e_{i}\right|^{r}\left|e_{i+k}-e_{i+k}^{*}\right|\left(\left|e_{i+k}\right|^{q-1}+\left|e_{i+k}^{*}\right|^{q-1}\right) \\
& +r\left|e_{i+k}^{*}\right|^{q}\left|e_{i}-e_{i}^{*}\right|\left(\left|e_{i}\right|^{r-1}+\left|e_{i}^{*}\right|^{r-1}\right) .
\end{aligned}
$$

Hence, by Minkowski's inequality, for any $p \geq 1$,

$$
\begin{aligned}
\left(E\left|e_{i}^{r} e_{i+k}^{q}-e_{i}^{* r} e_{i+k}^{* q}\right|^{p}\right)^{1 / p} \leq & q\left\{E\left[\left|e_{i}\right|^{r}\left|e_{i+k}-e_{i+k}^{*}\right|\left(\left|e_{i+k}\right|^{q-1}+\left|e_{i+k}^{*}\right|^{q-1}\right)\right]^{p}\right\}^{1 / p} \\
& +r\left\{E\left[\left|e_{i+k}^{*}\right|^{q}\left|e_{i}-e_{i}^{*}\right|\left(\left|e_{i}\right|^{r-1}+\left|e_{i}^{*}\right|^{r-1}\right)\right]^{p}\right\}^{1 / p} .
\end{aligned}
$$

Applying the Cauchy-Schwarz inequality to each term on the right-hand side, we get

$$
\begin{aligned}
& \left(E\left|e_{i}^{r} e_{i+k}^{q}-e_{i}^{* r} e_{i+k}^{* q}\right|^{p}\right)^{1 / p} \\
& \leq q\left\{E\left[\left|e_{i}\right|^{2 r p}\left(\left|e_{i+k}\right|^{q-1}+\left|e_{i+k}^{*}\right|^{q-1}\right)^{2 p}\right]\right\}^{1 /(2 p)}\left\{E\left|e_{i+k}-e_{i+k}^{*}\right|^{2 p}\right\}^{1 /(2 p)} \\
& +r\left\{E\left[\left|e_{i+k}^{*}\right|^{2 q p}\left(\left|e_{i}\right|^{r-1}+\left|e_{i}^{*}\right|^{r-1}\right)^{2 p}\right]\right\}^{1 /(2 p)}\left\{E\left|e_{i}-e_{i}^{*}\right|^{2 p}\right\}^{1 /(2 p)} .
\end{aligned}
$$

Since the $e_{i}$ and the $e_{i}^{*}$ have all moments and are elements of stationary fields, we conclude that

$$
\left(E\left|e_{i}^{r} e_{i+k}^{q}-e_{i}^{* r} e_{i+k}^{* q}\right|^{p}\right)^{1 / p} \leq C_{1}(r, q, p)\left\{E\left|e_{0}-e_{0}^{*}\right|^{2 p}\right\}^{1 /(2 p)} .
$$

We established that for all $k$ the process $e_{i}^{r} e_{i+k}^{q}$ is decomposable in the sense of El Machkouri et al. (2013). The claim thus follows from their Proposition 1.

The estimators based on the $z_{i}$ estimate the spatial long-run variances of the fields defined by

$$
f_{\mathcal{S}}\left(z_{i}\right)=z_{i}^{3}-3 z_{i} \quad \text { and } \quad f_{\mathcal{K}}\left(z_{i}\right)=z_{i}^{4}-6 z_{i}^{2}+3 .
$$

Observe that $f_{\mathcal{S}}$ and $f_{\mathcal{K}}$ are polynomials such that $E f_{\mathcal{S}}\left(z_{0}\right)=0$ and $E f_{\mathcal{K}}\left(z_{0}\right)=0$. This motivates the following lemma, which follows from Lemma II.6 by bounding all crossterms.

Lemma II.7 For the $z_{i}$ defined by (2.4) set $y_{i}=f\left(z_{i}\right)$, where $f$ is a polynomial such that $E y_{0}=0$. If Assumption 2.1 holds, then

$$
E\left|\sum_{i \in \Gamma}\left(y_{i} y_{i+k}-E\left[y_{0} y_{k}\right]\right)\right| \leq C(f)|\Gamma|^{1 / 2} .
$$

The next lemma established the consistency of the estimators based on the $z_{i}$.

Lemma II.8 Consider the estimator $\hat{\sigma}_{n}^{2}$ defined by (2.11) and the long-run variance $\sigma^{2}$ given by (2.9), both computed from $y_{i}=f\left(z_{i}\right)$, where $f$ is a polynomial such that $E y_{0}=0$. If Assumption 2.1 holds and $h^{\star}=o\left(n^{\star 1 / 2}\right)$, then $\hat{\sigma}_{n}^{2} \stackrel{P}{\rightarrow} \sigma^{2}$. 
Proof: We will denote by $C$ a positive constant, which may change from line to line. By Lemma II.7,

$$
\begin{aligned}
E\left|\sum_{j \in J(h)} w_{h}(j)(\hat{\gamma}(j)-\gamma(j))\right| & \leq\left.\sum_{j \in J(h)}\left|w_{h}(j)\right| E|| \Gamma_{n}(j)\right|^{-1} \sum_{i \in \Gamma_{n}(j)} y_{i} y_{i+j}-\gamma(j) \mid \\
& =\sum_{j \in J(h)}\left|w_{h}(j)\right| \frac{1}{\left|\Gamma_{n}(j)\right|} E\left|\sum_{i \in \Gamma_{n}(j)}\left[y_{i} y_{i+j}-\gamma(j)\right]\right| \\
& \leq C \sum_{j \in J(h)}\left|w_{h}(j)\right| \frac{1}{\left|\Gamma_{n}(j)\right|^{1 / 2}} .
\end{aligned}
$$

For the estimator (2.11),

$$
\sum_{j \in J(h)}\left|w_{h}(j)\right| \frac{1}{\left|\Gamma_{n}(j)\right|^{1 / 2}} \leq C \sum_{\left|j_{1}\right| \leq h_{1}} \ldots \sum_{\left|j_{d}\right| \leq m_{d}} \frac{1}{\left(n_{1} \ldots n_{d}\right)^{1 / 2}} \leq C \prod_{\ell=1}^{d}\left(\frac{h_{\ell}}{n_{\ell}^{1 / 2}}\right)^{d} .
$$

Recall that $h^{\star}=\max _{1 \leq \ell \leq d} h_{\ell}$ and $n^{\star}=\min _{1 \leq \ell \leq d} n_{\ell}$. Thus the condition $h^{\star}=o\left(n^{\star 1 / 2}\right)$ implies that for each $1 \leq \ell \leq d, h_{\ell} / n_{\ell}^{1 / 2} \rightarrow 0$, so we conclude that

$$
E\left|\hat{\sigma}_{n}^{2}-\sum_{j \in J(h)} w_{h}(j) \gamma(j)\right|=E\left|\sum_{j \in J(h)} w_{h}(j)(\hat{\gamma}(j)-\gamma(j))\right| \rightarrow 0 .
$$

It thus remains to check that $\sum_{j \in J(h)} w_{h}(j) \gamma(j) \rightarrow \sigma^{2}$, which reduces to $\sum_{j \notin J(h)} \gamma(j) \rightarrow 0$, which follows from $(2.10)$, and $\sum_{j \in J(h)}\left(1-w_{h}(j)\right) \gamma(j) \rightarrow 0$. The last relation can be explicitely written as

$$
\sum_{\ell=1}^{d} \sum_{\left|j_{\ell}\right| \leq n_{\ell}} \prod_{\ell=1}^{d}\left\{1-K\left(\frac{j_{\ell}}{n_{\ell}}\right)\right\} \gamma\left(j_{1}, \ldots, j_{d}\right) \rightarrow 0 .
$$

By Assumption 2.3, at least one $h_{\ell}$ tends to infinity, and for this $\ell, j_{\ell} / h_{\ell} \rightarrow 0$, for any $j_{\ell}$. Hence, for fixed $j_{1}, \ldots, j_{d}$, the product in (II.5) tends to 0. Relation (II.5) thus follows from Assumption 2.3, the summability condition (2.10), and dominated convergence.

Proof OF Theorem 2.2: We will verify only the convergence $\hat{\phi}_{\mathcal{S} \text {,kern }}^{2} \stackrel{P}{\rightarrow} \phi_{\mathcal{S}}^{2}$. The arguments for the second convergence are very similar; there are just more remainder terms. To lighten the notation, we will drop the $\mathcal{S}$, and use the subscripts $x$ and $z$ to indicate quatities computed, respectively, from the $x_{i}$ and the $z_{i}$. 
Recall the definitions of the sample mean $\bar{z}$ and variance $s_{z}^{2}$ given in (II.1), and observe that

$$
\begin{aligned}
y_{x, i}:=x_{i}^{3}-3 x_{i} & =\left(\frac{z_{i}-\bar{z}}{s_{z}}\right)^{3}-3 \frac{z_{i}-\bar{z}}{s_{z}} \\
& =\frac{1}{s_{z}^{3}}\left\{z_{i}^{3}-3 z_{i}^{2} \bar{z}+3 z_{i} \bar{z}^{2}-\bar{z}^{3}\right\}-\frac{3}{s_{z}} z_{i}+\frac{3}{s_{z}} \bar{z}
\end{aligned}
$$

which gives

$$
\bar{m}_{x}=\frac{1}{n_{\Gamma}} \sum_{i \in \Gamma_{n}} y_{x, i}=\frac{1}{s_{z}^{3}} \frac{1}{n_{\Gamma}} \sum_{i \in \Gamma_{n}} z_{i}^{3}-\frac{3}{s_{z}^{3}} \bar{z} \frac{1}{n_{\Gamma}} \sum_{i \in \Gamma_{n}} z_{i}^{2}+\frac{2}{s_{z}^{3}} \bar{z}^{3} .
$$

Since $y_{z, i}:=z_{i}^{3}-3 z_{i}$, we obtain $\bar{m}_{z}=n_{\Gamma}^{-1} \sum_{i \in \Gamma_{n}} z_{i}^{3}-3 \bar{z}$.

We will work with the decomposition

$$
\left(y_{x, i}-\bar{m}_{x}\right)\left(y_{x, i+j}-\bar{m}_{x}\right)=\left(y_{z, i}-\bar{m}_{z}\right)\left(y_{z, i+j}-\bar{m}_{z}\right)+R_{n}(i, j),
$$

where

$$
\begin{aligned}
R_{n}(i, j)= & \left(\left(y_{x, i}-y_{z, i}\right)-\left(\bar{m}_{x}-\bar{m}_{z}\right)\right)\left(y_{x, i+j}-\bar{m}_{x}\right) \\
& +\left(y_{z, i}-\bar{m}_{z}\right)\left(y_{x, i+j}-y_{z, i+j}-\left(\bar{m}_{x}-\bar{m}_{z}\right)\right) .
\end{aligned}
$$

By Lemma II.8,

$$
\sum_{j \in J(h)} w_{h}(j)\left|\Gamma_{n}(j)\right|^{-1} \sum_{i \in \Gamma_{n}(j)}\left(y_{z, i}-\bar{m}_{z}\right)\left(y_{z, i+j}-\bar{m}_{z}\right) \stackrel{P}{\rightarrow} \sigma_{z}^{2}=\phi_{\mathcal{S}}^{2}
$$

so to complete the proof we must show that

$$
\sum_{j \in J(h)} w_{h}(j)\left|\Gamma_{n}(j)\right|^{-1} \sum_{i \in \Gamma_{n}(j)} R_{n}(i, j) \stackrel{P}{\rightarrow} 0 .
$$

By (II.6), all quantities in $R_{n}(i, j)$ can be expressed in terms of the $z_{i}$. Then repeated applications of Lemma II.2 and convergence (II.3) establish (II.7). This completes the proof for the kernel estimators.

Turning to the power estimators, we only display the proof of $\hat{\phi}_{\mathcal{S} \text {,pow }}^{2} \stackrel{P}{\rightarrow} \phi_{\mathcal{S}}^{2}$. Observe that

$$
\begin{aligned}
\hat{\gamma}_{x}(j) & =\frac{1}{s_{z}^{2}} \frac{1}{\left|\Gamma_{n}(j)\right|} \sum_{i \in \Gamma_{n}(j)}\left(z_{i}-\bar{z}\right)\left(z_{i+j}-\bar{z}\right) \\
& =\frac{1}{s_{z}^{2}} \frac{1}{\left|\Gamma_{n}(j)\right|}\left\{\sum_{i \in \Gamma_{n}(j)} z_{i} z_{i+j}-\bar{z} \sum_{i \in \Gamma_{n}(j)} z_{i}-\bar{z} \sum_{i \in \Gamma_{n}(j)} z_{i+j}+\left|\Gamma_{n}(j)\right| \bar{z}^{2}\right\} .
\end{aligned}
$$


Proposition 1 of El Machkouri et al. (2013) implies that there is a constant $C$ such that

$$
E\left\{\sum_{i \in \Gamma_{n}(j)}\left(z_{i} z_{i+j}-E\left[z_{i} z_{i+j}\right]\right)\right\}^{2} \leq C\left|\Gamma_{n}(j)\right|,
$$

and

$$
E\left\{\sum_{i \in \Gamma_{n}(j)} z_{i}\right\}^{2} \leq C\left|\Gamma_{n}(j)\right|, \quad E\left\{\sum_{i \in \Gamma_{n}(j)} z_{i+j}\right\}^{2} \leq C\left|\Gamma_{n}(j)\right| .
$$

Since $\left|\hat{\gamma}_{x}(j)\right| \leq 1$ and $\rho_{j} \leq 1,\left|\hat{\gamma}_{x}^{3}(j)-\rho_{j}^{3}\right| \leq 3\left|\hat{\gamma}_{x}(j)-\rho_{j}\right|$ and therefore

$$
\begin{aligned}
\left|\sum_{j \in J(h)} w_{h}(j)\left[\hat{\gamma}_{x}^{3}(j)-\rho_{j}^{3}\right]\right| \leq & 3 \sum_{j \in J(h)}\left|w_{h}(j)\right|\left|\hat{\gamma}_{x}(j)-\rho_{j}\right| \\
\leq & 3 \sum_{j \in J(h)}\left|w_{h}(j)\right|\left|\frac{1}{s_{z}^{2}} \frac{1}{\left|\Gamma_{n}(j)\right|} \sum_{i \in \Gamma_{n}(j)} z_{i} z_{i+j}-\rho_{j}\right| \\
& +\frac{3}{s_{z}^{2}} O_{P}\left(\left|\Gamma_{n}(j)\right|^{-3 / 2}\right) \sum_{j \in J(h)} T_{n, j},
\end{aligned}
$$

with

$$
T_{n, j}=\left|w_{h}(j)\right|\left[\left|\sum_{i \in \Gamma_{n}(j)} z_{i}\right|+\left|\sum_{i \in \Gamma_{n}(j)} z_{i+j}\right|+\frac{1}{\left|\Gamma_{n}(j)\right|^{1 / 2}}\right] .
$$

By (II.9),

$$
E \sum_{j \in J(h)} T_{n, j}=O\left(\sum_{j \in J(h)} \frac{\left|w_{h}(j)\right|}{\left|\Gamma_{n}(j)\right|}\right)=O\left(\frac{h^{\star}}{n^{\star}}\right)=o(1) .
$$

To use (II.8) for the first term, we must separate the effect of $s_{z}^{2}$. Observe that

$$
\begin{aligned}
\sum_{j \in J(h)}\left|w_{h}(j)\right| & \left|\frac{1}{s_{z}^{2}} \frac{1}{\left|\Gamma_{n}(j)\right|} \sum_{i \in \Gamma_{n}(j)} z_{i} z_{i+j}-\rho_{j}\right| \\
\leq & \frac{1}{s_{z}^{2}} \sum_{j \in J(h)}\left|w_{h}(j)\right| \frac{1}{\left|\Gamma_{n}(j)\right|}\left|\sum_{i \in \Gamma_{n}(j)}\left[z_{i} z_{i+j}-\rho_{j}\right]\right| \\
& +\left|\frac{1}{s_{z}^{2}}-1\right| \sum_{j \in J(h)}\left|w_{h}(j)\right|\left|\rho_{j}\right|
\end{aligned}
$$

and

$$
\sum_{j \in J(h)}\left|w_{h}(j)\right| \frac{1}{\left|\Gamma_{n}(j)\right|}\left|\sum_{i \in \Gamma_{n}(j)}\left[z_{i} z_{i+j}-\rho_{j}\right]\right| \leq \sum_{j \in J(h)}\left|w_{h}(j)\right| \frac{\left|\Gamma_{n}(j)\right|^{1 / 2}}{\left|\Gamma_{n}(j)\right|}=o\left(\frac{h^{\star}}{n^{\star 1 / 2}}\right),
$$


by Assumption 2.3. Thus we get by Markov's inequality

$$
\hat{\phi}_{\mathcal{S}, \text { pow }}^{2}=6 \sum_{j \in J(h)} w_{h}(j) \rho_{j}^{3}+o_{P}(1) .
$$

By the assumption on the weights $w_{h}(j)$, the dominated convergence theorem yields $\sum_{j \in J(h)} w_{h}(j) \rho_{j}^{3} \rightarrow \sum_{j \in \mathbb{Z}^{d}} \rho_{j}^{3}$, completing the proof of the claim for the power estimators.

\section{II.3 Proofs of Theorems 2.3, 2.4 and Corollary 2.2}

Proof of TheOrem 2.3: We will show that $n_{\Gamma}^{-1 / 2} \mathcal{S}_{n}-E z_{0}^{3}=o_{P}(1)$. Verifications of the claims for $\mathcal{K}_{n}$ and $\mathcal{K}_{n}^{*}$ is similar, but longer because more terms are involved. They use relations derived in the proofs of Lemma II.4 and II.5 rather than relation (II.2) in the proof of Lemma II.3. Recall that Part 1) of Lemma II.2 holds under Asssumption 2.4 and it implies, in particular, (II.3), i.e. $s_{z}^{2} \stackrel{P}{\rightarrow} 1$, with the same justification.

We begin by rewriting relation (II.2), as

$$
\mathcal{S}_{n}=\frac{1}{n_{\Gamma}^{1 / 2} s_{z}^{3}} \sum_{i \in \Gamma_{n}}\left(z_{i}^{3}-3 z_{i}\right)-\frac{3}{n_{\Gamma}^{1 / 2} s_{z}^{3}} \bar{z} \sum_{i \in \Gamma_{n}}\left(z_{i}^{2}-1\right)+\frac{2}{n_{\Gamma}^{1 / 2} s_{z}^{3}} n_{\Gamma}^{3 / 2} \bar{z}^{3} .
$$

Using the above stated bounds, we see that

$$
\frac{1}{n_{\Gamma}^{1 / 2} s_{z}^{3}} \bar{z} \sum_{i \in \Gamma_{n}}\left(z_{i}^{2}-1\right)=O_{P}\left(n_{\Gamma}^{-1 / 2}\right)
$$

and

$$
\frac{1}{n_{\Gamma}^{1 / 2} s_{z}^{3}} n_{\Gamma}^{3 / 2} \bar{z}^{3}=O_{P}\left(n_{\Gamma}^{-1 / 2}\right)
$$

Therefore,

$$
\mathcal{S}_{n}=\frac{1}{n_{\Gamma}^{1 / 2} s_{z}^{3}} \sum_{i \in \Gamma_{n}}\left(z_{i}^{3}-3 z_{i}\right)+O_{P}\left(n_{\Gamma}^{-1 / 2}\right)
$$

Next, observe that

$$
\begin{aligned}
\mathcal{S}_{n}-n_{\Gamma}^{1 / 2} E z_{0}^{3} & =\frac{1}{n_{\Gamma}^{1 / 2} s_{z}^{3}} \sum_{i \in \Gamma_{n}}\left(z_{i}^{3}-3 z_{i}\right)-n_{\Gamma}^{1 / 2} E z_{0}^{3}+O_{P}\left(n_{\Gamma}^{-1 / 2}\right) \\
& =\frac{1}{n_{\Gamma}^{1 / 2} s_{z}^{3}} \sum_{i \in \Gamma_{n}}\left(z_{i}^{3}-3 z_{i}-E z_{0}^{3}\right)+n_{\Gamma}^{1 / 2}\left(1-s_{z}^{3}\right) \frac{1}{s_{z}^{3}} E z_{0}^{3}+O_{P}\left(n_{\Gamma}^{-1 / 2}\right) .
\end{aligned}
$$

We must show that $n_{\Gamma}^{-1 / 2}\left(\mathcal{S}_{n}-n_{\Gamma}^{1 / 2} E z_{0}^{3}\right)=o_{P}(1)$. By Theorem 1 of El Machkouri et al. (2013), $n_{\Gamma}^{-1 / 2} \sum_{i \in \Gamma_{n}}\left(z_{i}^{3}-3 z_{i}-E z_{0}^{3}\right)$ is asymptotically normal with mean zero. Using (II.3), we see that

$$
n_{\Gamma}^{-1 / 2}\left(\mathcal{S}_{n}-n_{\Gamma}^{1 / 2} E z_{0}^{3}\right)=O_{P}\left(n_{\Gamma}^{-1 / 2}\right)+o_{P}(1)+O_{P}\left(n_{\Gamma}^{-1}\right)
$$


completing the proof.

Proof of Theorem 2.4: To prove the claims for the kernel estimators, one must follow the proof of Theorem 2.2. However, since the $Z_{i}$ might not be normally distributed, we must replace the bound $\sum_{i \in \Gamma_{n}} z_{i}^{3}=O_{p}\left(n_{\Gamma}^{1 / 2}\right)$ with $\sum_{i \in \Gamma_{n}} z_{i}^{3}=O_{p}\left(n_{\Gamma}\right)$. Hence (II.7) will be replaced with

$$
\sum_{j \in J(h)} w_{h}(j)\left|\Gamma_{n}(j)\right|^{-1} \sum_{i \in \Gamma_{n}(j)} R_{n}(i, j) o_{P}\left(h^{\star}\right)
$$

To establish the claim for the power estimator, we also follow the corresponding part of the proof of Theorem 2.2. Inequalities (II.8) and (II.9) continue to hold under Assumption 2.4, and following the remaining steps we see that $\hat{\phi}_{\mathcal{S} \text {,pow }}^{2} \stackrel{P}{\rightarrow} 6 \sum_{i \in \mathbb{Z}^{d}} \rho_{i}^{3}$, establishing the claim for the skewness. The claims for the kurtosis can be verified in a similar manner, and so are omitted.

Proof of Corollary 2.2: We consider first the statistics based on the kernel estimators, for which the argument is a bit longer. Suppose $E z_{0}^{3} \neq 0$ and consider the factorization

$$
\frac{\mathcal{S}_{n}^{2}}{\hat{\phi}_{\mathcal{S}, \text { kern }}^{2}}=\left(\frac{\mathcal{S}_{n}}{n_{\Gamma}^{1 / 2}}\right)^{2}\left(\frac{h^{\star}}{\hat{\phi}_{\mathcal{S}, \text { kern }}^{2}}\right) \frac{n_{\Gamma}}{h^{\star}} .
$$

According to Theorem 2.3, the first factor converges to $E z_{0}^{3}$. By the first part of Theorem 2.4, for all $\delta>0$, there are $M$ and $n_{0}$ such that $P\left\{\hat{\phi}_{\mathcal{S}, \text {,ern }} / h^{\star} \leq M\right\} \geq 1-\delta$ if $n_{\Gamma} \geq n_{0}$, i.e.

$$
P\left\{\frac{h^{\star}}{\hat{\phi}_{\mathcal{S}, \text { kern }}^{2}} \geq \frac{1}{M}\right\} \geq 1-\delta, \quad \text { if } n_{\Gamma} \geq n_{0} .
$$

The assumption $h^{\star}=o\left(n^{\star 1 / 2}\right)$ implies that $n_{\Gamma} / h^{\star} \rightarrow \infty$. We thus conclude that (II.10) diverges to infinity in probability. Basically the same argument shows that if $E z_{0}^{4} \neq 3$, then $\mathcal{K}_{n}^{2} / \hat{\phi}_{\mathcal{K} \text {,kern }}^{2}$ diverges. For the power estimators, we must replace $h^{\star}$ in (II.10) by 1 , and argue in the same way.

\section{Explicit formulas for a 2D domain}

We observe a realization $\left\{X_{i, j}, 1 \leq i \leq N, 1 \leq j \leq N\right\}$ of a stationary random field on $\mathbb{Z} \times \mathbb{Z}$. We want to test if the field is Gaussian. We work with studentized observations

$$
x_{i, j}=\frac{X_{i, j}-\bar{X}}{S}
$$

where

$$
\bar{X}=\frac{1}{N M} \sum_{i=1}^{N} \sum_{j=1}^{M} X_{i, j}, \quad S^{2}=\frac{1}{N M} \sum_{i=1}^{N} \sum_{j=1}^{M}\left(X_{i, j}-\bar{X}\right)^{2}
$$


The modified sample skewness and kurtosis are defined by

$$
\mathcal{S}^{\star}=\frac{1}{\sqrt{N M}} \sum_{i=1}^{N} \sum_{j=1}^{M}\left(x_{i, j}^{3}-3 x_{i, j}\right) \quad \text { and } \quad \mathcal{K}^{\star}=\frac{1}{\sqrt{N M}} \sum_{i=1}^{N} \sum_{j=1}^{M}\left(x_{i, j}^{4}-6 x_{i, j}^{2}+3\right)
$$

and the test statistic by

$$
J^{\star}:=\frac{\mathcal{S}^{\star 2}}{\hat{\phi}_{\mathcal{S}}^{2}}+\frac{\mathcal{K}^{\star 2}}{\hat{\phi}_{\mathcal{K}}^{2}},
$$

where $\hat{\phi}_{\mathcal{S}}^{2}$ and $\hat{\phi}_{\mathcal{K}}^{2}$ are consistent estimators of the spatial long-run variances $\phi_{\mathcal{S}}^{2}$ and $\phi_{\mathcal{K}}^{2}$, which we now specify.

Kernel estimators These are the estimators given by (2.12). Set

$$
y_{i, j}^{(\mathcal{S})}=x_{i, j}^{3}-3 x_{i, j} \quad \text { and } \quad y_{i, j}^{(\mathcal{K})}=x_{i, j}^{4}-6 x_{i, j}^{2}+3 .
$$

For generic observations $y_{i, j}$ define the sample mean

$$
\bar{y}=\frac{1}{N M} \sum_{i=1}^{N} \sum_{j=1}^{M} y_{i, j}
$$

and the sample covariances

$$
\hat{\gamma}(u, v)=\frac{1}{|D(u, v)|} \sum_{i, j \in D(u, v)}\left(y_{i, j}-\bar{y}\right)\left(y_{i+u, j+v}-\bar{y}\right)
$$

where $D(u, v)$ is the subset of the grid such that $1 \leq i, i+u \leq N$ and $1 \leq j, j+v \leq M$, and and $|D(u, v)|$ is its cardinality. Then $\phi_{\mathcal{S}}^{2}$ and $\phi_{\mathcal{K}}^{2}$ are computed as

$$
\hat{\sigma}_{\text {kern }}^{2}=\sum_{|u| \leq N} \sum_{|v| \leq M} K\left(\frac{u}{h}\right) K\left(\frac{v}{h}\right) \hat{\gamma}(u, v)
$$

with $y_{i, j}=y_{i, j}^{(\mathcal{S})}$ or $y_{i, j}=y_{i, j}^{(\mathcal{K})}$.

Power estimators These are the estimators given by (2.13). The sample covariances $\hat{\gamma}_{x}(u, v)$ are computed from the $x_{i, j}$ given by (III.1) using formula (III.2) with $y_{i, j}$ replaced by $x_{i, j}$. Then

$$
\hat{\phi}_{\mathcal{S}}^{2}=6 \sum_{|u| \leq N} \sum_{|v| \leq N} K\left(\frac{u}{h}\right)\left(\frac{v}{h}\right) \hat{\gamma}_{x}^{3}(u, v), \quad \hat{\phi}_{\mathcal{K}}^{2}=24 \sum_{|u| \leq N} \sum_{|v| \leq N} K\left(\frac{u}{h}\right) K\left(\frac{v}{h}\right) \hat{\gamma}_{x}^{4}(u, v) .
$$

\section{Empirical power under skew-normal distribution}

This section presents the simulation results of empirical power when the three DGPs in Section 3.1 are generated by $\xi_{i, j}$ with i.i.d. skew-normal distribution. 
Treated in Azzalini (2014), the skew-normal distribution is denoted as $\mathcal{S} \mathcal{N}\left(\eta, \omega^{2}, \alpha\right)$, where $\eta$ is the location parameter, $\omega$ is the scale parameter, and $\alpha$ is shape parameter. The skew-normal distribution has the property that an increasing value of its shape parameter, $\alpha$, induces a larger skewness. Thus, we vary the numerical value of $\alpha$ from 1 to 3 in order to examine the sensitivity of our test. To mitigate the impact from the other two parameters, the scale parameter $\omega$ is always chosen to be 1 and the location parameter $\eta$ is set to be the value that ensure the mean of the distribution is zero, i.e. $\eta=-\omega \frac{\alpha}{\sqrt{1+\alpha^{2}}} \sqrt{\frac{2}{\pi}}$. Table 8 shows the theoretical skewness and kurtosis for the chosen sets of the parameters. As can be seen, the skew-normal skewness of the distribution is small when $\alpha=1$, and the distribution becomes moderately skewed when $\alpha=3$.

Table 8: Theoretical skewness and kurtosis of the skew-normal distribution

\begin{tabular}{lccc}
\hline \hline$\left(\eta, \omega^{2}, \alpha\right)$ & $(-0.564,1,1)$ & $(-0.714,1,2)$ & $(-0.757,1,3)$ \\
\hline skewness $\approx$ & 0.137 & 0.454 & 0.667 \\
kurtosis $\approx$ & 3.062 & 3.305 & 3.510 \\
\hline \hline
\end{tabular}

Tables 9 and 10 present the empirical power of the test for DGPs with skew-normal distribution by $5 \%$ significance level critical values with the long-run variance estimator choice of the kernel estimator and the power estimator, respectively. We have several observations in the following. Firstly, the power increase with a larger grid size $N$, as we expected. Secondly, the power increase with the growth in the value of the shape parameter $\alpha$. Although the skewness is small when $\alpha=1$, the test can achieve high empirical power when the grid size $N=100$. The power is almost $100 \%$ if the shape parameter $\alpha$ is larger, such as 2 and 3. Thirdly, there is no obvious difference in the empirical power when use the kernel estimator or the power estimator for the long-run variance. Lastly, comparing different kernel functions, the Bartlett kernel occasionally produces a marginally higher power, but this may due to the slight inflation in its sizes which can be observed in Table 1.

\section{V $\quad \mathbf{P}$-values for January, April, and October anomalies}

Tables 11 to 16 present the $\mathrm{P}$-values of the normality tests applied to monthly SST anomalies in the months of January, April and October in all years of the sample period, which is between September 1981 to December 2014. Definitions of the anomalies $D_{i, j}(t)$ and $U_{i, j}(t)$, together with other details, are given in Section 3.2. 
Table 9: Empirical power of the DGPs with skew-normal distribution - kernel estimator

\begin{tabular}{|c|c|c|c|c|}
\hline \multicolumn{5}{|c|}{ Panel A: Spatial IID } \\
\hline Grid Size & Kernel & $\alpha=1$ & $\alpha=2$ & $\alpha=3$ \\
\hline $\mathrm{N}=25$ & $\begin{array}{l}\text { Truncated } \\
\text { Bartlett } \\
\text { Flat-top }\end{array}$ & $\begin{array}{l}29.42 \% \\
20.44 \% \\
22.78 \% \\
\end{array}$ & $\begin{array}{l}97.16 \% \\
98.06 \% \\
97.80 \% \\
\end{array}$ & $\begin{array}{r}99.84 \% \\
99.96 \% \\
100.00 \% \\
\end{array}$ \\
\hline $\mathrm{N}=50$ & $\begin{array}{l}\text { Truncated } \\
\text { Bartlett } \\
\text { Flat-top }\end{array}$ & $\begin{array}{l}69.86 \% \\
68.98 \% \\
69.18 \% \\
\end{array}$ & $\begin{array}{l}100.00 \% \\
100.00 \% \\
100.00 \% \\
\end{array}$ & $\begin{array}{l}100.00 \% \\
100.00 \% \\
100.00 \% \\
\end{array}$ \\
\hline $\mathrm{N}=100$ & $\begin{array}{l}\text { Truncated } \\
\text { Bartlett } \\
\text { Flat-top }\end{array}$ & $\begin{array}{l}99.94 \% \\
99.94 \% \\
99.92 \%\end{array}$ & $\begin{array}{l}100.00 \% \\
100.00 \% \\
100.00 \%\end{array}$ & $\begin{array}{l}100.00 \% \\
100.00 \% \\
100.00 \%\end{array}$ \\
\hline \multicolumn{5}{|c|}{ Panel B: Spatial Moving Average } \\
\hline Grid Size & Kernel & $\alpha=1$ & $\alpha=2$ & $\alpha=3$ \\
\hline $\mathrm{N}=25$ & $\begin{array}{l}\text { Truncated } \\
\text { Bartlett } \\
\text { Flat-top }\end{array}$ & $\begin{array}{l}22.52 \% \\
15.26 \% \\
16.08 \% \\
\end{array}$ & $\begin{array}{l}85.14 \% \\
86.42 \% \\
85.06 \% \\
\end{array}$ & $\begin{array}{l}99.08 \% \\
99.72 \% \\
99.32 \% \\
\end{array}$ \\
\hline $\mathbf{N}=50$ & $\begin{array}{l}\text { Truncated } \\
\text { Bartlett } \\
\text { Flat-top }\end{array}$ & $\begin{array}{l}46.32 \% \\
46.14 \% \\
44.74 \%\end{array}$ & $\begin{array}{l}100.00 \% \\
100.00 \% \\
100.00 \%\end{array}$ & $\begin{array}{l}100.00 \% \\
100.00 \% \\
100.00 \%\end{array}$ \\
\hline $\mathbf{N}=100$ & $\begin{array}{l}\text { Truncated } \\
\text { Bartlett } \\
\text { Flat-top }\end{array}$ & $\begin{array}{l}96.96 \% \\
97.18 \% \\
97.00 \% \\
\end{array}$ & $\begin{array}{l}100.00 \% \\
100.00 \% \\
100.00 \%\end{array}$ & $\begin{array}{l}100.00 \% \\
100.00 \% \\
100.00 \%\end{array}$ \\
\hline \multicolumn{5}{|c|}{ Panel C: Spatial Autoregressive } \\
\hline Grid Size & Kernel & $\alpha=1$ & $\alpha=2$ & $\alpha=3$ \\
\hline $\mathrm{N}=25$ & $\begin{array}{l}\text { Truncated } \\
\text { Bartlett } \\
\text { Flat-top }\end{array}$ & $\begin{array}{l}18.50 \% \\
15.02 \% \\
13.36 \%\end{array}$ & $\begin{array}{l}76.62 \% \\
80.64 \% \\
75.08 \%\end{array}$ & $\begin{array}{l}96.72 \% \\
99.06 \% \\
97.94 \%\end{array}$ \\
\hline $\mathrm{N}=50$ & $\begin{array}{l}\text { Truncated } \\
\text { Bartlett } \\
\text { Flat-top }\end{array}$ & $\begin{array}{l}36.32 \% \\
40.36 \% \\
35.42 \% \\
\end{array}$ & $\begin{array}{l}100.00 \% \\
100.00 \% \\
100.00 \% \\
\end{array}$ & $\begin{array}{l}100.00 \% \\
100.00 \% \\
100.00 \% \\
\end{array}$ \\
\hline $\mathrm{N}=100$ & $\begin{array}{l}\text { Truncated } \\
\text { Bartlett } \\
\text { Flat-top }\end{array}$ & $\begin{array}{l}91.34 \% \\
93.04 \% \\
91.48 \%\end{array}$ & $\begin{array}{l}100.00 \% \\
100.00 \% \\
100.00 \%\end{array}$ & $\begin{array}{l}100.00 \% \\
100.00 \% \\
100.00 \%\end{array}$ \\
\hline
\end{tabular}


Table 10: Empirical power of the DGPs with skew-normal distribution - power estimator

\begin{tabular}{|c|c|c|c|c|}
\hline \multicolumn{5}{|c|}{ Panel A: Spatial IID } \\
\hline Grid Size & Kernel & $\alpha=1$ & $\alpha=2$ & $\alpha=3$ \\
\hline \multirow{3}{*}{$\mathrm{N}=25$} & Truncated & $22.00 \%$ & $98.38 \%$ & $100.00 \%$ \\
\hline & Bartlett & $22.02 \%$ & $98.40 \%$ & $100.00 \%$ \\
\hline & Flat-top & $22.02 \%$ & $98.40 \%$ & $100.00 \%$ \\
\hline \multirow{3}{*}{$\mathrm{N}=50$} & Truncated & $70.28 \%$ & $100.00 \%$ & $100.00 \%$ \\
\hline & Bartlett & $70.28 \%$ & $100.00 \%$ & $100.00 \%$ \\
\hline & Flat-top & $70.28 \%$ & $100.00 \%$ & $100.00 \%$ \\
\hline \multirow{3}{*}{$\mathbf{N}=100$} & Truncated & $99.94 \%$ & $100.00 \%$ & $100.00 \%$ \\
\hline & Bartlett & $99.94 \%$ & $100.00 \%$ & $100.00 \%$ \\
\hline & Flat-top & $99.94 \%$ & $100.00 \%$ & $100.00 \%$ \\
\hline \multicolumn{5}{|c|}{ Panel B: Spatial Moving Average } \\
\hline Grid Size & Kernel & $\alpha=1$ & $\alpha=2$ & $\alpha=3$ \\
\hline \multirow{3}{*}{$\mathrm{N}=25$} & Truncated & $14.04 \%$ & $87.32 \%$ & $99.74 \%$ \\
\hline & Bartlett & $15.44 \%$ & $89.04 \%$ & $99.80 \%$ \\
\hline & Flat-top & $13.98 \%$ & $87.26 \%$ & $99.74 \%$ \\
\hline \multirow{3}{*}{$\mathrm{N}=50$} & Truncated & $46.28 \%$ & $100.00 \%$ & $100.00 \%$ \\
\hline & Bartlett & $48.06 \%$ & $100.00 \%$ & $100.00 \%$ \\
\hline & Flat-top & $46.28 \%$ & $100.00 \%$ & $100.00 \%$ \\
\hline \multirow{3}{*}{$\mathrm{N}=100$} & Truncated & $96.98 \%$ & $100.00 \%$ & $100.00 \%$ \\
\hline & Bartlett & $97.26 \%$ & $100.00 \%$ & $100.00 \%$ \\
\hline & Flat-top & $96.98 \%$ & $100.00 \%$ & $100.00 \%$ \\
\hline \multicolumn{5}{|c|}{ Panel C: Spatial Autoregressive } \\
\hline Grid Size & Kernel & $\alpha=1$ & $\alpha=2$ & $\alpha=3$ \\
\hline \multirow{3}{*}{$\mathrm{N}=25$} & Truncated & $11.20 \%$ & $73.30 \%$ & $98.58 \%$ \\
\hline & Bartlett & $15.08 \%$ & $79.62 \%$ & $99.30 \%$ \\
\hline & Flat-top & $11.62 \%$ & $73.82 \%$ & $98.66 \%$ \\
\hline \multirow{3}{*}{$\mathrm{N}=50$} & Truncated & $35.52 \%$ & $99.96 \%$ & $100.00 \%$ \\
\hline & Bartlett & $41.58 \%$ & $99.98 \%$ & $100.00 \%$ \\
\hline & Flat-top & $36.48 \%$ & $99.98 \%$ & $100.00 \%$ \\
\hline \multirow{3}{*}{$\mathrm{N}=100$} & Truncated & $91.48 \%$ & $100.00 \%$ & $100.00 \%$ \\
\hline & Bartlett & $93.72 \%$ & $100.00 \%$ & $100.00 \%$ \\
\hline & Flat-top & $91.52 \%$ & $100.00 \%$ & $100.00 \%$ \\
\hline
\end{tabular}


Table 11: P-values of the spatial normality test for $D_{i, j}(t)$ in January.

\begin{tabular}{|c|c|c|c|c|c|c|c|c|}
\hline \multirow[b]{2}{*}{ Date } & \multicolumn{4}{|c|}{ Kernel Estimator } & \multicolumn{4}{|c|}{ Power Estimator } \\
\hline & Region 1 & Region 2 & Region 3 & Region 4 & Region 1 & Region 2 & Region 3 & Region 4 \\
\hline Jan-1982 & $0.4 \%$ & $0.0 \%$ & $0.0 \%$ & $0.1 \%$ & $0.3 \%$ & $0.2 \%$ & $0.1 \%$ & $0.0 \%$ \\
\hline Jan-1983 & $0.0 \%$ & $0.0 \%$ & $0.0 \%$ & $1.3 \%$ & $0.0 \%$ & $0.0 \%$ & $0.8 \%$ & $0.5 \%$ \\
\hline Jan-1984 & $0.7 \%$ & $0.0 \%$ & $0.0 \%$ & $43.4 \%$ & $13.1 \%$ & $0.0 \%$ & $0.2 \%$ & $0.0 \%$ \\
\hline Jan-1985 & $0.0 \%$ & $0.0 \%$ & $0.2 \%$ & $0.0 \%$ & $1.0 \%$ & $0.0 \%$ & $5.8 \%$ & $0.0 \%$ \\
\hline Jan-1986 & $0.6 \%$ & $0.0 \%$ & $0.0 \%$ & $0.0 \%$ & $5.5 \%$ & $0.0 \%$ & $0.7 \%$ & $0.0 \%$ \\
\hline Jan-1987 & $15.1 \%$ & $0.0 \%$ & $0.0 \%$ & $0.1 \%$ & $39.1 \%$ & $0.0 \%$ & $0.0 \%$ & $0.0 \%$ \\
\hline Jan-1988 & $0.0 \%$ & $0.0 \%$ & $0.0 \%$ & $0.0 \%$ & $0.0 \%$ & $0.0 \%$ & $0.4 \%$ & $0.0 \%$ \\
\hline Jan-1989 & $86.1 \%$ & $0.0 \%$ & $0.0 \%$ & $45.3 \%$ & $87.2 \%$ & $0.0 \%$ & $0.0 \%$ & $45.0 \%$ \\
\hline Jan-1990 & $0.0 \%$ & $46.9 \%$ & $70.5 \%$ & $0.0 \%$ & $0.0 \%$ & $60.1 \%$ & $77.2 \%$ & $0.0 \%$ \\
\hline Jan-1991 & $75.1 \%$ & $0.0 \%$ & $0.0 \%$ & $6.7 \%$ & $82.4 \%$ & $0.0 \%$ & $0.0 \%$ & $0.0 \%$ \\
\hline Jan-1992 & $0.0 \%$ & $0.0 \%$ & $0.0 \%$ & $11.2 \%$ & $0.0 \%$ & $0.0 \%$ & $0.3 \%$ & $14.7 \%$ \\
\hline Jan-1993 & $0.0 \%$ & $0.0 \%$ & $0.0 \%$ & $0.0 \%$ & $0.0 \%$ & $0.0 \%$ & $0.2 \%$ & $0.0 \%$ \\
\hline Jan-1994 & $0.0 \%$ & $0.0 \%$ & $0.0 \%$ & $0.0 \%$ & $0.1 \%$ & $0.0 \%$ & $0.0 \%$ & $0.0 \%$ \\
\hline Jan-1995 & $0.0 \%$ & $0.0 \%$ & $0.6 \%$ & $0.0 \%$ & $0.0 \%$ & $0.0 \%$ & $0.3 \%$ & $0.0 \%$ \\
\hline Jan-1996 & $54.9 \%$ & $0.0 \%$ & $2.4 \%$ & $0.0 \%$ & $53.9 \%$ & $0.0 \%$ & $12.1 \%$ & $0.0 \%$ \\
\hline Jan-1997 & $26.5 \%$ & $7.8 \%$ & $0.0 \%$ & $0.0 \%$ & $55.1 \%$ & $30.1 \%$ & $0.0 \%$ & $0.0 \%$ \\
\hline Jan-1998 & $0.0 \%$ & $0.0 \%$ & $0.0 \%$ & $0.0 \%$ & $0.0 \%$ & $0.0 \%$ & $1.0 \%$ & $0.0 \%$ \\
\hline Jan-1999 & $0.0 \%$ & $0.0 \%$ & $0.0 \%$ & $0.0 \%$ & $0.3 \%$ & $0.0 \%$ & $0.0 \%$ & $0.0 \%$ \\
\hline Jan-2000 & $0.0 \%$ & $0.0 \%$ & $0.0 \%$ & $0.0 \%$ & $0.0 \%$ & $0.0 \%$ & $3.8 \%$ & $0.0 \%$ \\
\hline Jan-2001 & $15.6 \%$ & $0.2 \%$ & $0.0 \%$ & $86.1 \%$ & $44.8 \%$ & $6.5 \%$ & $0.9 \%$ & $0.2 \%$ \\
\hline Jan-2002 & $0.0 \%$ & $0.0 \%$ & $0.1 \%$ & $0.0 \%$ & $0.0 \%$ & $0.0 \%$ & $0.1 \%$ & $0.0 \%$ \\
\hline Jan-2003 & $0.0 \%$ & $0.0 \%$ & $0.1 \%$ & $17.2 \%$ & $0.0 \%$ & $0.0 \%$ & $0.3 \%$ & $4.3 \%$ \\
\hline Jan-2004 & $0.3 \%$ & $0.0 \%$ & $0.0 \%$ & $0.0 \%$ & $8.4 \%$ & $0.4 \%$ & $3.1 \%$ & $0.0 \%$ \\
\hline Jan-2005 & $2.1 \%$ & $21.2 \%$ & $2.8 \%$ & $6.5 \%$ & $14.3 \%$ & $34.3 \%$ & $20.7 \%$ & $6.8 \%$ \\
\hline Jan-2006 & $6.6 \%$ & $0.0 \%$ & $0.0 \%$ & $0.0 \%$ & $18.3 \%$ & $0.0 \%$ & $0.0 \%$ & $1.6 \%$ \\
\hline Jan-2007 & $6.1 \%$ & $19.3 \%$ & $0.0 \%$ & $7.5 \%$ & $13.8 \%$ & $51.5 \%$ & $0.0 \%$ & $0.0 \%$ \\
\hline Jan-2008 & $0.1 \%$ & $0.0 \%$ & $0.0 \%$ & $17.7 \%$ & $3.6 \%$ & $0.0 \%$ & $0.0 \%$ & $0.0 \%$ \\
\hline Jan-2009 & $0.0 \%$ & $0.0 \%$ & $0.0 \%$ & $41.5 \%$ & $0.0 \%$ & $0.0 \%$ & $2.0 \%$ & $0.0 \%$ \\
\hline Jan-2010 & $32.3 \%$ & $0.0 \%$ & $0.0 \%$ & $0.0 \%$ & $9.9 \%$ & $0.0 \%$ & $0.0 \%$ & $0.0 \%$ \\
\hline Jan-2011 & $0.0 \%$ & $0.0 \%$ & $0.0 \%$ & $0.0 \%$ & $0.0 \%$ & $0.0 \%$ & $0.0 \%$ & $0.0 \%$ \\
\hline Jan-2012 & $0.0 \%$ & $0.0 \%$ & $0.8 \%$ & $1.9 \%$ & $0.0 \%$ & $1.4 \%$ & $12.9 \%$ & $0.1 \%$ \\
\hline Jan-2013 & $0.0 \%$ & $19.0 \%$ & $0.0 \%$ & $0.0 \%$ & $0.0 \%$ & $28.7 \%$ & $0.1 \%$ & $0.0 \%$ \\
\hline Jan-2014 & $0.0 \%$ & $0.0 \%$ & $1.0 \%$ & $0.1 \%$ & $0.0 \%$ & $0.0 \%$ & $15.2 \%$ & $0.0 \%$ \\
\hline
\end{tabular}


Table 12: P-values of the spatial normality test for $D_{i, j}(t)$ in April.

\begin{tabular}{|c|c|c|c|c|c|c|c|c|}
\hline \multirow[b]{2}{*}{ Date } & \multicolumn{4}{|c|}{ Kernel Estimator } & \multicolumn{4}{|c|}{ Power Estimator } \\
\hline & Region 1 & Region 2 & Region 3 & Region 4 & Region 1 & Region 2 & Region 3 & Region 4 \\
\hline Apr-1982 & $0.0 \%$ & $0.0 \%$ & $52.6 \%$ & $0.6 \%$ & $0.0 \%$ & $0.0 \%$ & $44.3 \%$ & $0.0 \%$ \\
\hline Apr-1983 & $0.0 \%$ & $0.0 \%$ & $0.0 \%$ & $0.0 \%$ & $0.0 \%$ & $0.0 \%$ & $0.0 \%$ & $0.4 \%$ \\
\hline Apr-1984 & $0.2 \%$ & $1.4 \%$ & $0.0 \%$ & $0.2 \%$ & $0.1 \%$ & $11.2 \%$ & $0.0 \%$ & $0.0 \%$ \\
\hline Apr-1985 & $0.0 \%$ & $0.0 \%$ & $0.0 \%$ & $5.5 \%$ & $0.0 \%$ & $0.0 \%$ & $0.0 \%$ & $11.4 \%$ \\
\hline Apr-1986 & $8.5 \%$ & $0.1 \%$ & $0.2 \%$ & $0.0 \%$ & $10.6 \%$ & $4.7 \%$ & $5.7 \%$ & $0.0 \%$ \\
\hline Apr-1987 & $2.4 \%$ & $0.0 \%$ & $0.0 \%$ & $0.0 \%$ & $0.0 \%$ & $0.0 \%$ & $0.0 \%$ & $0.0 \%$ \\
\hline Apr-1988 & $35.8 \%$ & $0.0 \%$ & $0.0 \%$ & $0.0 \%$ & $38.5 \%$ & $0.0 \%$ & $0.7 \%$ & $0.0 \%$ \\
\hline Apr-1989 & $4.2 \%$ & $0.0 \%$ & $0.0 \%$ & $0.0 \%$ & $0.0 \%$ & $0.5 \%$ & $0.0 \%$ & $0.0 \%$ \\
\hline Apr-1990 & $5.9 \%$ & $0.0 \%$ & $43.7 \%$ & $47.6 \%$ & $0.2 \%$ & $0.0 \%$ & $37.0 \%$ & $33.8 \%$ \\
\hline Apr-1991 & $11.9 \%$ & $1.9 \%$ & $0.0 \%$ & $7.9 \%$ & $31.4 \%$ & $0.0 \%$ & $0.0 \%$ & $0.0 \%$ \\
\hline Apr-1992 & $11.0 \%$ & $0.0 \%$ & $0.0 \%$ & $0.0 \%$ & $16.5 \%$ & $0.0 \%$ & $0.0 \%$ & $0.0 \%$ \\
\hline Apr-1993 & $1.3 \%$ & $0.0 \%$ & $72.6 \%$ & $5.3 \%$ & $0.0 \%$ & $1.5 \%$ & $82.9 \%$ & $0.0 \%$ \\
\hline Apr-1994 & $0.0 \%$ & $0.0 \%$ & $0.0 \%$ & $0.0 \%$ & $3.3 \%$ & $0.1 \%$ & $0.0 \%$ & $0.0 \%$ \\
\hline Apr-1995 & $0.1 \%$ & $0.0 \%$ & $38.1 \%$ & $0.0 \%$ & $0.0 \%$ & $0.1 \%$ & $57.9 \%$ & $0.0 \%$ \\
\hline Apr-1996 & $1.2 \%$ & $0.0 \%$ & $0.7 \%$ & $66.6 \%$ & $14.2 \%$ & $3.3 \%$ & $0.7 \%$ & $82.4 \%$ \\
\hline Apr-1997 & $0.0 \%$ & $0.0 \%$ & $0.1 \%$ & $0.0 \%$ & $0.1 \%$ & $0.0 \%$ & $1.3 \%$ & $0.4 \%$ \\
\hline Apr-1998 & $0.0 \%$ & $0.0 \%$ & $0.0 \%$ & $0.0 \%$ & $0.1 \%$ & $0.6 \%$ & $0.0 \%$ & $0.0 \%$ \\
\hline Apr-1999 & $7.6 \%$ & $0.0 \%$ & $0.0 \%$ & $0.1 \%$ & $7.4 \%$ & $0.0 \%$ & $0.0 \%$ & $3.9 \%$ \\
\hline Apr-2000 & $0.0 \%$ & $0.0 \%$ & $0.1 \%$ & $0.0 \%$ & $0.7 \%$ & $0.0 \%$ & $5.2 \%$ & $0.0 \%$ \\
\hline Apr-2001 & $2.4 \%$ & $0.0 \%$ & $0.0 \%$ & $0.0 \%$ & $3.3 \%$ & $0.8 \%$ & $0.0 \%$ & $1.8 \%$ \\
\hline Apr-2002 & $55.3 \%$ & $9.1 \%$ & $0.4 \%$ & $10.2 \%$ & $57.6 \%$ & $1.6 \%$ & $3.2 \%$ & $11.4 \%$ \\
\hline Apr-2003 & $0.0 \%$ & $0.8 \%$ & $0.2 \%$ & $0.9 \%$ & $0.0 \%$ & $8.1 \%$ & $1.4 \%$ & $8.1 \%$ \\
\hline Apr-2004 & $0.5 \%$ & $77.9 \%$ & $0.0 \%$ & $51.3 \%$ & $4.6 \%$ & $83.8 \%$ & $0.0 \%$ & $5.0 \%$ \\
\hline Apr-2005 & $2.3 \%$ & $12.3 \%$ & $5.2 \%$ & $0.0 \%$ & $0.0 \%$ & $28.6 \%$ & $17.6 \%$ & $0.0 \%$ \\
\hline Apr-2006 & $0.0 \%$ & $0.7 \%$ & $36.6 \%$ & $11.1 \%$ & $0.0 \%$ & $3.5 \%$ & $59.4 \%$ & $0.0 \%$ \\
\hline Apr-2007 & $0.0 \%$ & $1.6 \%$ & $0.0 \%$ & $0.0 \%$ & $0.0 \%$ & $6.0 \%$ & $0.6 \%$ & $0.0 \%$ \\
\hline Apr-2008 & $0.0 \%$ & $0.0 \%$ & $0.0 \%$ & $19.8 \%$ & $0.0 \%$ & $2.0 \%$ & $0.6 \%$ & $0.0 \%$ \\
\hline Apr-2009 & $0.0 \%$ & $3.7 \%$ & $0.0 \%$ & $13.3 \%$ & $0.0 \%$ & $11.7 \%$ & $0.3 \%$ & $0.0 \%$ \\
\hline Apr-2010 & $0.0 \%$ & $0.0 \%$ & $3.5 \%$ & $0.0 \%$ & $0.0 \%$ & $0.1 \%$ & $24.8 \%$ & $0.0 \%$ \\
\hline Apr-2011 & $0.0 \%$ & $0.0 \%$ & $0.0 \%$ & $0.0 \%$ & $0.0 \%$ & $0.0 \%$ & $0.1 \%$ & $2.1 \%$ \\
\hline Apr-2012 & $3.8 \%$ & $10.4 \%$ & $0.0 \%$ & $4.6 \%$ & $14.9 \%$ & $23.3 \%$ & $0.0 \%$ & $7.1 \%$ \\
\hline Apr-2013 & $0.1 \%$ & $4.1 \%$ & $24.6 \%$ & $0.0 \%$ & $0.2 \%$ & $6.4 \%$ & $30.5 \%$ & $0.0 \%$ \\
\hline Apr-2014 & $0.0 \%$ & $3.7 \%$ & $0.0 \%$ & $0.0 \%$ & $0.0 \%$ & $15.4 \%$ & $1.0 \%$ & $0.0 \%$ \\
\hline
\end{tabular}


Table 13: P-values of the spatial normality test for $D_{i, j}(t)$ in October.

\begin{tabular}{|c|c|c|c|c|c|c|c|c|}
\hline \multirow[b]{2}{*}{ Date } & \multicolumn{4}{|c|}{ Kernel Estimator } & \multicolumn{4}{|c|}{ Power Estimator } \\
\hline & Region 1 & Region 2 & Region 3 & Region 4 & Region 1 & Region 2 & Region 3 & Region 4 \\
\hline Oct-1981 & $0.8 \%$ & $0.5 \%$ & $42.6 \%$ & $0.0 \%$ & $2.7 \%$ & $0.0 \%$ & $57.9 \%$ & $0.1 \%$ \\
\hline Oct-1982 & $3.9 \%$ & $0.0 \%$ & $3.7 \%$ & $0.0 \%$ & $2.9 \%$ & $0.0 \%$ & $8.0 \%$ & $0.0 \%$ \\
\hline Oct-1983 & $0.1 \%$ & $0.0 \%$ & $0.0 \%$ & $22.7 \%$ & $5.4 \%$ & $0.0 \%$ & $0.0 \%$ & $14.5 \%$ \\
\hline Oct-1984 & $20.8 \%$ & $6.5 \%$ & $2.8 \%$ & $2.7 \%$ & $4.8 \%$ & $22.0 \%$ & $11.3 \%$ & $18.7 \%$ \\
\hline Oct-1985 & $0.0 \%$ & $8.0 \%$ & $0.3 \%$ & $0.0 \%$ & $0.0 \%$ & $23.3 \%$ & $0.1 \%$ & $0.0 \%$ \\
\hline Oct-1986 & $0.0 \%$ & $0.1 \%$ & $11.7 \%$ & $0.0 \%$ & $3.6 \%$ & $4.8 \%$ & $3.4 \%$ & $0.0 \%$ \\
\hline Oct-1987 & $21.3 \%$ & $0.0 \%$ & $0.0 \%$ & $0.0 \%$ & $45.9 \%$ & $0.1 \%$ & $1.0 \%$ & $0.0 \%$ \\
\hline Oct-1988 & $0.0 \%$ & $0.0 \%$ & $0.0 \%$ & $0.0 \%$ & $0.0 \%$ & $0.0 \%$ & $0.0 \%$ & $0.0 \%$ \\
\hline Oct-1989 & $38.5 \%$ & $0.0 \%$ & $2.0 \%$ & $15.4 \%$ & $45.2 \%$ & $0.0 \%$ & $16.1 \%$ & $19.5 \%$ \\
\hline Oct-1990 & $5.8 \%$ & $0.1 \%$ & $0.2 \%$ & $5.2 \%$ & $16.9 \%$ & $1.9 \%$ & $0.5 \%$ & $12.7 \%$ \\
\hline Oct-1991 & $0.0 \%$ & $0.0 \%$ & $0.0 \%$ & $2.4 \%$ & $0.0 \%$ & $1.1 \%$ & $0.0 \%$ & $2.4 \%$ \\
\hline Oct-1992 & $1.4 \%$ & $0.0 \%$ & $0.0 \%$ & $0.0 \%$ & $1.2 \%$ & $0.0 \%$ & $0.2 \%$ & $0.0 \%$ \\
\hline Oct-1993 & $2.0 \%$ & $0.0 \%$ & $0.0 \%$ & $5.2 \%$ & $2.2 \%$ & $0.0 \%$ & $1.1 \%$ & $0.4 \%$ \\
\hline Oct-1994 & $0.0 \%$ & $0.0 \%$ & $0.0 \%$ & $0.0 \%$ & $0.0 \%$ & $0.0 \%$ & $1.7 \%$ & $0.0 \%$ \\
\hline Oct-1995 & $0.1 \%$ & $0.0 \%$ & $30.5 \%$ & $0.0 \%$ & $0.0 \%$ & $0.0 \%$ & $38.2 \%$ & $0.0 \%$ \\
\hline Oct-1996 & $0.0 \%$ & $0.0 \%$ & $0.0 \%$ & $0.0 \%$ & $0.0 \%$ & $0.0 \%$ & $0.2 \%$ & $0.0 \%$ \\
\hline Oct-1997 & $2.1 \%$ & $0.0 \%$ & $4.4 \%$ & $0.0 \%$ & $0.0 \%$ & $0.0 \%$ & $23.8 \%$ & $0.0 \%$ \\
\hline Oct-1998 & $0.0 \%$ & $0.0 \%$ & $0.0 \%$ & $0.0 \%$ & $1.0 \%$ & $0.0 \%$ & $0.0 \%$ & $0.0 \%$ \\
\hline Oct-1999 & $28.1 \%$ & $0.0 \%$ & $0.0 \%$ & $0.0 \%$ & $27.9 \%$ & $0.0 \%$ & $0.0 \%$ & $0.0 \%$ \\
\hline Oct-2000 & $23.8 \%$ & $0.0 \%$ & $3.0 \%$ & $0.0 \%$ & $3.0 \%$ & $0.2 \%$ & $3.1 \%$ & $0.0 \%$ \\
\hline Oct-2001 & $69.1 \%$ & $17.0 \%$ & $0.1 \%$ & $17.6 \%$ & $80.8 \%$ & $44.4 \%$ & $2.2 \%$ & $5.7 \%$ \\
\hline Oct-2002 & $8.2 \%$ & $0.0 \%$ & $0.0 \%$ & $0.0 \%$ & $13.8 \%$ & $0.0 \%$ & $1.7 \%$ & $0.0 \%$ \\
\hline Oct-2003 & $0.1 \%$ & $28.1 \%$ & $2.2 \%$ & $0.0 \%$ & $4.1 \%$ & $26.1 \%$ & $0.0 \%$ & $0.0 \%$ \\
\hline Oct-2004 & $0.2 \%$ & $0.0 \%$ & $0.0 \%$ & $0.0 \%$ & $0.0 \%$ & $3.0 \%$ & $0.0 \%$ & $0.0 \%$ \\
\hline Oct-2005 & $0.1 \%$ & $0.3 \%$ & $0.0 \%$ & $20.5 \%$ & $0.0 \%$ & $5.2 \%$ & $0.0 \%$ & $25.6 \%$ \\
\hline Oct-2006 & $0.0 \%$ & $0.0 \%$ & $0.0 \%$ & $0.0 \%$ & $0.7 \%$ & $0.0 \%$ & $0.0 \%$ & $0.0 \%$ \\
\hline Oct-2007 & $0.2 \%$ & $1.0 \%$ & $0.0 \%$ & $16.2 \%$ & $0.7 \%$ & $3.2 \%$ & $0.0 \%$ & $0.0 \%$ \\
\hline Oct-2008 & $0.0 \%$ & $0.0 \%$ & $19.0 \%$ & $0.0 \%$ & $0.5 \%$ & $0.0 \%$ & $30.7 \%$ & $0.1 \%$ \\
\hline Oct-2009 & $42.2 \%$ & $0.0 \%$ & $1.5 \%$ & $0.0 \%$ & $49.7 \%$ & $0.3 \%$ & $15.0 \%$ & $0.0 \%$ \\
\hline Oct-2010 & $0.2 \%$ & $0.0 \%$ & $0.2 \%$ & $12.5 \%$ & $0.8 \%$ & $0.0 \%$ & $0.6 \%$ & $0.0 \%$ \\
\hline Oct-2011 & $25.6 \%$ & $0.0 \%$ & $0.7 \%$ & $0.5 \%$ & $24.7 \%$ & $0.0 \%$ & $0.3 \%$ & $0.0 \%$ \\
\hline Oct-2012 & $34.8 \%$ & $0.0 \%$ & $0.0 \%$ & $0.0 \%$ & $38.0 \%$ & $0.3 \%$ & $0.0 \%$ & $0.0 \%$ \\
\hline Oct-2013 & $0.9 \%$ & $0.1 \%$ & $0.0 \%$ & $0.0 \%$ & $0.3 \%$ & $0.1 \%$ & $0.0 \%$ & $0.0 \%$ \\
\hline Oct-2014 & $22.8 \%$ & $0.0 \%$ & $58.4 \%$ & $0.1 \%$ & $45.5 \%$ & $0.3 \%$ & $71.0 \%$ & $0.0 \%$ \\
\hline
\end{tabular}


Table 14: P-values of the spatial normality test for $U_{i, j}(t)$ in January.

\begin{tabular}{|c|c|c|c|c|c|c|c|c|}
\hline \multirow[b]{2}{*}{ Date } & \multicolumn{4}{|c|}{ Kernel Estimator } & \multicolumn{4}{|c|}{ Power Estimator } \\
\hline & Region 1 & Region 2 & Region 3 & Region 4 & Region 1 & Region 2 & Region 3 & Region 4 \\
\hline Jan-1982 & $1.6 \%$ & $1.2 \%$ & $0.0 \%$ & $5.1 \%$ & $2.1 \%$ & $12.6 \%$ & $0.1 \%$ & $9.0 \%$ \\
\hline Jan-1983 & $0.0 \%$ & $0.0 \%$ & $0.0 \%$ & $2.7 \%$ & $0.0 \%$ & $0.0 \%$ & $0.1 \%$ & $6.6 \%$ \\
\hline Jan-1984 & $0.0 \%$ & $0.0 \%$ & $0.0 \%$ & $15.2 \%$ & $0.1 \%$ & $0.0 \%$ & $0.1 \%$ & $14.5 \%$ \\
\hline Jan-1985 & $0.1 \%$ & $0.0 \%$ & $60.6 \%$ & $0.0 \%$ & $3.3 \%$ & $0.0 \%$ & $74.9 \%$ & $0.0 \%$ \\
\hline Jan-1986 & $0.0 \%$ & $0.4 \%$ & $0.0 \%$ & $0.0 \%$ & $0.0 \%$ & $7.8 \%$ & $1.4 \%$ & $0.0 \%$ \\
\hline Jan-1987 & $3.3 \%$ & $0.0 \%$ & $0.0 \%$ & $0.2 \%$ & $8.7 \%$ & $0.1 \%$ & $0.0 \%$ & $0.0 \%$ \\
\hline Jan-1988 & $0.0 \%$ & $0.0 \%$ & $3.3 \%$ & $0.0 \%$ & $0.0 \%$ & $0.0 \%$ & $21.6 \%$ & $0.0 \%$ \\
\hline Jan-1989 & $0.0 \%$ & $0.0 \%$ & $0.0 \%$ & $0.0 \%$ & $0.0 \%$ & $0.0 \%$ & $0.0 \%$ & $0.7 \%$ \\
\hline Jan-1990 & $0.0 \%$ & $2.0 \%$ & $5.9 \%$ & $3.3 \%$ & $2.6 \%$ & $13.1 \%$ & $18.1 \%$ & $4.2 \%$ \\
\hline Jan-1991 & $0.1 \%$ & $0.4 \%$ & $0.0 \%$ & $2.8 \%$ & $2.3 \%$ & $0.0 \%$ & $2.1 \%$ & $1.1 \%$ \\
\hline Jan-1992 & $0.1 \%$ & $0.0 \%$ & $0.0 \%$ & $0.0 \%$ & $5.5 \%$ & $2.2 \%$ & $1.6 \%$ & $0.0 \%$ \\
\hline Jan-1993 & $0.0 \%$ & $0.0 \%$ & $0.0 \%$ & $2.7 \%$ & $0.0 \%$ & $0.0 \%$ & $0.0 \%$ & $9.3 \%$ \\
\hline Jan-1994 & $0.0 \%$ & $0.4 \%$ & $0.0 \%$ & $0.0 \%$ & $0.0 \%$ & $9.9 \%$ & $0.0 \%$ & $0.0 \%$ \\
\hline Jan-1995 & $3.1 \%$ & $0.0 \%$ & $0.0 \%$ & $0.0 \%$ & $23.1 \%$ & $0.0 \%$ & $0.0 \%$ & $0.0 \%$ \\
\hline Jan-1996 & $0.0 \%$ & $0.0 \%$ & $20.6 \%$ & $0.0 \%$ & $0.0 \%$ & $0.1 \%$ & $39.8 \%$ & $0.0 \%$ \\
\hline Jan-1997 & $0.0 \%$ & $50.5 \%$ & $0.0 \%$ & $0.0 \%$ & $0.0 \%$ & $65.1 \%$ & $0.2 \%$ & $0.0 \%$ \\
\hline Jan-1998 & $0.0 \%$ & $0.0 \%$ & $0.0 \%$ & $0.0 \%$ & $0.0 \%$ & $0.5 \%$ & $2.1 \%$ & $0.0 \%$ \\
\hline Jan-1999 & $0.0 \%$ & $0.0 \%$ & $0.0 \%$ & $0.0 \%$ & $0.1 \%$ & $0.0 \%$ & $0.0 \%$ & $0.0 \%$ \\
\hline Jan-2000 & $0.0 \%$ & $0.4 \%$ & $0.2 \%$ & $0.0 \%$ & $0.1 \%$ & $2.1 \%$ & $6.9 \%$ & $0.0 \%$ \\
\hline Jan-2001 & $0.0 \%$ & $0.0 \%$ & $0.0 \%$ & $0.0 \%$ & $0.0 \%$ & $0.0 \%$ & $0.1 \%$ & $0.0 \%$ \\
\hline Jan-2002 & $0.0 \%$ & $0.0 \%$ & $1.8 \%$ & $0.0 \%$ & $0.0 \%$ & $0.9 \%$ & $2.1 \%$ & $0.0 \%$ \\
\hline Jan-2003 & $0.0 \%$ & $0.0 \%$ & $41.8 \%$ & $0.0 \%$ & $0.0 \%$ & $2.0 \%$ & $64.6 \%$ & $0.1 \%$ \\
\hline Jan-2004 & $0.0 \%$ & $0.0 \%$ & $0.0 \%$ & $0.0 \%$ & $0.0 \%$ & $0.4 \%$ & $0.0 \%$ & $0.0 \%$ \\
\hline Jan-2005 & $14.3 \%$ & $36.3 \%$ & $6.9 \%$ & $0.0 \%$ & $14.3 \%$ & $27.4 \%$ & $32.2 \%$ & $0.0 \%$ \\
\hline Jan-2006 & $31.3 \%$ & $0.0 \%$ & $0.0 \%$ & $0.0 \%$ & $51.4 \%$ & $0.0 \%$ & $0.0 \%$ & $0.0 \%$ \\
\hline Jan-2007 & $11.6 \%$ & $7.0 \%$ & $0.0 \%$ & $0.0 \%$ & $22.3 \%$ & $10.9 \%$ & $0.1 \%$ & $0.0 \%$ \\
\hline Jan-2008 & $0.0 \%$ & $0.0 \%$ & $0.0 \%$ & $53.8 \%$ & $1.8 \%$ & $0.0 \%$ & $0.0 \%$ & $11.1 \%$ \\
\hline Jan-2009 & $0.0 \%$ & $0.0 \%$ & $0.0 \%$ & $55.0 \%$ & $0.0 \%$ & $0.0 \%$ & $0.0 \%$ & $60.7 \%$ \\
\hline Jan-2010 & $46.3 \%$ & $2.2 \%$ & $0.0 \%$ & $0.0 \%$ & $58.8 \%$ & $1.5 \%$ & $0.0 \%$ & $0.0 \%$ \\
\hline Jan-2011 & $0.0 \%$ & $0.0 \%$ & $0.0 \%$ & $0.0 \%$ & $0.5 \%$ & $0.0 \%$ & $0.1 \%$ & $0.0 \%$ \\
\hline Jan-2012 & $62.3 \%$ & $0.0 \%$ & $0.0 \%$ & $1.6 \%$ & $76.0 \%$ & $0.0 \%$ & $0.4 \%$ & $5.5 \%$ \\
\hline Jan-2013 & $10.4 \%$ & $0.0 \%$ & $0.0 \%$ & $1.4 \%$ & $0.6 \%$ & $0.0 \%$ & $0.1 \%$ & $4.3 \%$ \\
\hline Jan-2014 & $0.0 \%$ & $0.0 \%$ & $0.0 \%$ & $0.0 \%$ & $0.0 \%$ & $0.0 \%$ & $1.2 \%$ & $0.0 \%$ \\
\hline
\end{tabular}


Table 15: P-values of the spatial normality test for $U_{i, j}(t)$ in April.

\begin{tabular}{|c|c|c|c|c|c|c|c|c|}
\hline \multirow[b]{2}{*}{ Date } & \multicolumn{4}{|c|}{ Kernel Estimator } & \multicolumn{4}{|c|}{ Power Estimator } \\
\hline & Region 1 & Region 2 & Region 3 & Region 4 & Region 1 & Region 2 & Region 3 & Region 4 \\
\hline Apr-1982 & $0.0 \%$ & $0.0 \%$ & $7.5 \%$ & $1.2 \%$ & $0.2 \%$ & $0.0 \%$ & $26.5 \%$ & $0.0 \%$ \\
\hline Apr-1983 & $0.0 \%$ & $0.0 \%$ & $0.0 \%$ & $0.0 \%$ & $0.0 \%$ & $0.0 \%$ & $0.1 \%$ & $0.4 \%$ \\
\hline Apr-1984 & $0.0 \%$ & $0.0 \%$ & $0.0 \%$ & $9.2 \%$ & $0.1 \%$ & $0.0 \%$ & $0.0 \%$ & $16.8 \%$ \\
\hline Apr-1985 & $15.3 \%$ & $95.0 \%$ & $0.0 \%$ & $0.0 \%$ & $40.8 \%$ & $95.0 \%$ & $0.6 \%$ & $0.0 \%$ \\
\hline Apr-1986 & $13.0 \%$ & $0.0 \%$ & $0.1 \%$ & $0.0 \%$ & $32.5 \%$ & $0.0 \%$ & $1.3 \%$ & $0.0 \%$ \\
\hline Apr-1987 & $5.7 \%$ & $0.0 \%$ & $0.0 \%$ & $0.0 \%$ & $0.9 \%$ & $0.0 \%$ & $0.2 \%$ & $0.0 \%$ \\
\hline Apr-1988 & $92.7 \%$ & $0.0 \%$ & $0.0 \%$ & $0.0 \%$ & $95.7 \%$ & $0.0 \%$ & $0.3 \%$ & $0.0 \%$ \\
\hline Apr-1989 & $32.2 \%$ & $0.0 \%$ & $0.0 \%$ & $0.0 \%$ & $23.8 \%$ & $0.0 \%$ & $0.0 \%$ & $0.0 \%$ \\
\hline Apr-1990 & $25.3 \%$ & $0.0 \%$ & $15.9 \%$ & $0.0 \%$ & $35.3 \%$ & $0.0 \%$ & $28.2 \%$ & $0.0 \%$ \\
\hline Apr-1991 & $5.6 \%$ & $7.4 \%$ & $0.0 \%$ & $0.0 \%$ & $6.1 \%$ & $10.4 \%$ & $0.0 \%$ & $0.0 \%$ \\
\hline Apr-1992 & $1.2 \%$ & $0.0 \%$ & $0.0 \%$ & $0.0 \%$ & $1.8 \%$ & $0.3 \%$ & $0.0 \%$ & $0.0 \%$ \\
\hline Apr-1993 & $4.9 \%$ & $0.0 \%$ & $21.1 \%$ & $7.0 \%$ & $17.7 \%$ & $0.0 \%$ & $43.0 \%$ & $0.0 \%$ \\
\hline Apr-1994 & $0.0 \%$ & $1.0 \%$ & $0.0 \%$ & $0.0 \%$ & $0.1 \%$ & $17.4 \%$ & $2.7 \%$ & $0.0 \%$ \\
\hline Apr-1995 & $44.6 \%$ & $0.0 \%$ & $0.0 \%$ & $0.0 \%$ & $35.2 \%$ & $1.4 \%$ & $1.9 \%$ & $0.0 \%$ \\
\hline Apr-1996 & $0.0 \%$ & $0.0 \%$ & $45.7 \%$ & $10.6 \%$ & $3.5 \%$ & $0.0 \%$ & $69.7 \%$ & $16.6 \%$ \\
\hline Apr-1997 & $0.0 \%$ & $0.0 \%$ & $0.0 \%$ & $0.0 \%$ & $1.6 \%$ & $0.0 \%$ & $0.1 \%$ & $0.0 \%$ \\
\hline Apr-1998 & $0.0 \%$ & $0.0 \%$ & $0.0 \%$ & $0.0 \%$ & $0.0 \%$ & $0.0 \%$ & $0.0 \%$ & $0.0 \%$ \\
\hline Apr-1999 & $4.0 \%$ & $0.0 \%$ & $0.0 \%$ & $0.0 \%$ & $22.9 \%$ & $0.0 \%$ & $0.0 \%$ & $0.0 \%$ \\
\hline Apr-2000 & $0.0 \%$ & $0.0 \%$ & $0.0 \%$ & $0.0 \%$ & $1.7 \%$ & $0.0 \%$ & $4.4 \%$ & $0.0 \%$ \\
\hline Apr-2001 & $33.1 \%$ & $0.0 \%$ & $0.0 \%$ & $0.0 \%$ & $36.3 \%$ & $0.0 \%$ & $1.0 \%$ & $0.1 \%$ \\
\hline Apr-2002 & $1.4 \%$ & $0.0 \%$ & $0.0 \%$ & $0.0 \%$ & $14.8 \%$ & $0.0 \%$ & $0.2 \%$ & $5.7 \%$ \\
\hline Apr-2003 & $0.0 \%$ & $0.0 \%$ & $5.3 \%$ & $0.0 \%$ & $0.0 \%$ & $0.4 \%$ & $2.9 \%$ & $0.6 \%$ \\
\hline Apr-2004 & $0.0 \%$ & $0.0 \%$ & $0.0 \%$ & $3.6 \%$ & $0.2 \%$ & $0.0 \%$ & $0.0 \%$ & $6.8 \%$ \\
\hline Apr-2005 & $0.0 \%$ & $9.2 \%$ & $0.0 \%$ & $0.0 \%$ & $0.0 \%$ & $6.2 \%$ & $0.2 \%$ & $0.0 \%$ \\
\hline Apr-2006 & $0.0 \%$ & $0.0 \%$ & $23.5 \%$ & $1.8 \%$ & $0.3 \%$ & $0.0 \%$ & $45.7 \%$ & $0.0 \%$ \\
\hline Apr-2007 & $0.0 \%$ & $0.0 \%$ & $0.0 \%$ & $0.0 \%$ & $0.0 \%$ & $0.7 \%$ & $0.0 \%$ & $0.0 \%$ \\
\hline Apr-2008 & $0.0 \%$ & $0.0 \%$ & $0.0 \%$ & $31.3 \%$ & $0.0 \%$ & $0.0 \%$ & $0.0 \%$ & $0.0 \%$ \\
\hline Apr-2009 & $0.0 \%$ & $1.1 \%$ & $0.0 \%$ & $82.2 \%$ & $0.0 \%$ & $10.6 \%$ & $0.2 \%$ & $79.5 \%$ \\
\hline Apr-2010 & $0.0 \%$ & $0.0 \%$ & $0.0 \%$ & $0.0 \%$ & $0.0 \%$ & $0.0 \%$ & $0.3 \%$ & $0.0 \%$ \\
\hline Apr-2011 & $0.0 \%$ & $0.0 \%$ & $0.0 \%$ & $0.0 \%$ & $0.9 \%$ & $0.0 \%$ & $0.0 \%$ & $0.0 \%$ \\
\hline Apr-2012 & $38.5 \%$ & $0.0 \%$ & $0.0 \%$ & $0.0 \%$ & $53.8 \%$ & $0.1 \%$ & $0.0 \%$ & $0.7 \%$ \\
\hline Apr-2013 & $20.0 \%$ & $0.0 \%$ & $0.2 \%$ & $0.0 \%$ & $31.9 \%$ & $3.7 \%$ & $0.4 \%$ & $0.2 \%$ \\
\hline Apr-2014 & $0.0 \%$ & $14.8 \%$ & $0.0 \%$ & $0.0 \%$ & $0.0 \%$ & $45.7 \%$ & $0.1 \%$ & $0.0 \%$ \\
\hline
\end{tabular}


Table 16: P-values of the spatial normality test for $U_{i, j}(t)$ in October.

\begin{tabular}{|c|c|c|c|c|c|c|c|c|}
\hline \multirow[b]{2}{*}{ Date } & \multicolumn{4}{|c|}{ Kernel Estimator } & \multicolumn{4}{|c|}{ Power Estimator } \\
\hline & Region 1 & Region 2 & Region 3 & Region 4 & Region 1 & Region 2 & Region 3 & Region 4 \\
\hline Oct-1981 & $0.0 \%$ & $0.8 \%$ & $20.7 \%$ & $14.2 \%$ & $1.6 \%$ & $0.0 \%$ & $28.3 \%$ & $42.3 \%$ \\
\hline Oct-1982 & $67.5 \%$ & $0.0 \%$ & $0.0 \%$ & $0.0 \%$ & $74.1 \%$ & $0.4 \%$ & $0.0 \%$ & $0.0 \%$ \\
\hline Oct-1983 & $0.0 \%$ & $0.0 \%$ & $0.0 \%$ & $63.3 \%$ & $0.2 \%$ & $0.0 \%$ & $0.0 \%$ & $72.5 \%$ \\
\hline Oct-1984 & $51.1 \%$ & $0.0 \%$ & $0.0 \%$ & $0.0 \%$ & $51.7 \%$ & $0.0 \%$ & $1.8 \%$ & $0.0 \%$ \\
\hline Oct-1985 & $14.1 \%$ & $0.1 \%$ & $51.3 \%$ & $0.1 \%$ & $30.3 \%$ & $0.0 \%$ & $53.1 \%$ & $5.7 \%$ \\
\hline Oct-1986 & $0.0 \%$ & $0.0 \%$ & $48.2 \%$ & $6.3 \%$ & $0.5 \%$ & $0.0 \%$ & $49.5 \%$ & $20.9 \%$ \\
\hline Oct-1987 & $11.4 \%$ & $0.0 \%$ & $8.7 \%$ & $0.0 \%$ & $33.3 \%$ & $0.0 \%$ & $18.4 \%$ & $0.0 \%$ \\
\hline Oct-1988 & $0.0 \%$ & $0.0 \%$ & $0.0 \%$ & $0.0 \%$ & $0.2 \%$ & $0.0 \%$ & $0.7 \%$ & $0.0 \%$ \\
\hline Oct-1989 & $5.7 \%$ & $2.0 \%$ & $0.0 \%$ & $1.1 \%$ & $2.4 \%$ & $8.8 \%$ & $0.0 \%$ & $9.5 \%$ \\
\hline Oct-1990 & $0.0 \%$ & $1.6 \%$ & $0.0 \%$ & $0.0 \%$ & $0.3 \%$ & $20.3 \%$ & $0.0 \%$ & $0.3 \%$ \\
\hline Oct-1991 & $11.3 \%$ & $0.0 \%$ & $0.0 \%$ & $0.0 \%$ & $11.1 \%$ & $0.0 \%$ & $0.0 \%$ & $2.2 \%$ \\
\hline Oct-1992 & $13.9 \%$ & $0.0 \%$ & $0.0 \%$ & $29.6 \%$ & $42.3 \%$ & $0.1 \%$ & $0.5 \%$ & $2.6 \%$ \\
\hline Oct-1993 & $0.6 \%$ & $0.0 \%$ & $0.0 \%$ & $0.5 \%$ & $3.6 \%$ & $0.0 \%$ & $1.7 \%$ & $0.4 \%$ \\
\hline Oct-1994 & $0.0 \%$ & $0.0 \%$ & $0.0 \%$ & $0.0 \%$ & $0.0 \%$ & $0.0 \%$ & $0.0 \%$ & $0.0 \%$ \\
\hline Oct-1995 & $0.0 \%$ & $0.0 \%$ & $11.4 \%$ & $0.0 \%$ & $0.0 \%$ & $0.0 \%$ & $36.2 \%$ & $0.0 \%$ \\
\hline Oct-1996 & $0.0 \%$ & $0.0 \%$ & $0.0 \%$ & $0.0 \%$ & $0.0 \%$ & $0.0 \%$ & $0.0 \%$ & $0.0 \%$ \\
\hline Oct-1997 & $0.0 \%$ & $1.3 \%$ & $32.5 \%$ & $0.0 \%$ & $0.0 \%$ & $14.0 \%$ & $42.7 \%$ & $0.0 \%$ \\
\hline Oct-1998 & $0.0 \%$ & $0.0 \%$ & $0.0 \%$ & $0.0 \%$ & $0.0 \%$ & $0.1 \%$ & $0.0 \%$ & $0.0 \%$ \\
\hline Oct-1999 & $1.5 \%$ & $0.0 \%$ & $0.0 \%$ & $0.0 \%$ & $2.3 \%$ & $0.3 \%$ & $0.0 \%$ & $0.7 \%$ \\
\hline Oct-2000 & $35.9 \%$ & $0.0 \%$ & $5.7 \%$ & $0.0 \%$ & $59.5 \%$ & $0.3 \%$ & $12.2 \%$ & $0.0 \%$ \\
\hline Oct-2001 & $26.4 \%$ & $0.0 \%$ & $0.0 \%$ & $1.0 \%$ & $55.5 \%$ & $0.0 \%$ & $0.8 \%$ & $11.0 \%$ \\
\hline Oct-2002 & $6.0 \%$ & $0.0 \%$ & $0.0 \%$ & $0.0 \%$ & $9.3 \%$ & $0.1 \%$ & $0.3 \%$ & $0.0 \%$ \\
\hline Oct-2003 & $0.0 \%$ & $5.9 \%$ & $1.6 \%$ & $0.0 \%$ & $0.0 \%$ & $0.0 \%$ & $0.2 \%$ & $0.0 \%$ \\
\hline Oct-2004 & $50.4 \%$ & $2.6 \%$ & $0.0 \%$ & $0.0 \%$ & $57.0 \%$ & $1.7 \%$ & $0.0 \%$ & $0.0 \%$ \\
\hline Oct-2005 & $0.3 \%$ & $0.0 \%$ & $1.0 \%$ & $0.1 \%$ & $0.0 \%$ & $0.0 \%$ & $4.6 \%$ & $3.4 \%$ \\
\hline Oct-2006 & $0.0 \%$ & $0.0 \%$ & $0.0 \%$ & $0.0 \%$ & $0.1 \%$ & $0.0 \%$ & $0.0 \%$ & $0.0 \%$ \\
\hline Oct-2007 & $0.4 \%$ & $0.0 \%$ & $3.1 \%$ & $32.3 \%$ & $2.9 \%$ & $0.0 \%$ & $3.8 \%$ & $0.0 \%$ \\
\hline Oct-2008 & $0.0 \%$ & $0.0 \%$ & $0.5 \%$ & $0.0 \%$ & $2.6 \%$ & $0.0 \%$ & $5.9 \%$ & $0.4 \%$ \\
\hline Oct-2009 & $0.5 \%$ & $0.0 \%$ & $0.0 \%$ & $0.0 \%$ & $7.7 \%$ & $0.0 \%$ & $0.6 \%$ & $0.0 \%$ \\
\hline Oct-2010 & $63.7 \%$ & $0.0 \%$ & $0.0 \%$ & $0.1 \%$ & $68.8 \%$ & $0.0 \%$ & $0.1 \%$ & $0.1 \%$ \\
\hline Oct-2011 & $0.6 \%$ & $0.0 \%$ & $50.8 \%$ & $2.2 \%$ & $4.2 \%$ & $0.0 \%$ & $54.0 \%$ & $0.0 \%$ \\
\hline Oct-2012 & $0.0 \%$ & $0.0 \%$ & $0.0 \%$ & $0.0 \%$ & $0.0 \%$ & $1.1 \%$ & $0.0 \%$ & $0.0 \%$ \\
\hline Oct-2013 & $3.1 \%$ & $0.8 \%$ & $32.5 \%$ & $0.1 \%$ & $21.5 \%$ & $0.0 \%$ & $50.7 \%$ & $0.0 \%$ \\
\hline Oct-2014 & $4.5 \%$ & $0.0 \%$ & $0.0 \%$ & $0.0 \%$ & $11.3 \%$ & $0.1 \%$ & $0.8 \%$ & $0.0 \%$ \\
\hline
\end{tabular}

